\title{
11. SEDIMENT DISTRIBUTION AND DEPOSITIONAL PROCESSES OPERATING IN THE LESSER ANTILLES INTRAOCEANIC ISLAND ARC, EASTERN CARIBBEAN1
}

\author{
Audrey Wright, Earth Sciences and Marine Studies, University of California at Santa Cruz ${ }^{2}$
}

\begin{abstract}
Data from piston cores, DSDP drill sites, and seismic profiles in the Lesser Antilles intraoceanic island arc define eight lithofacies units: (I) a pelagic clay facies on the abyssal plain below the CCD (carbonate compensation depth); (II) a marly calcareous ooze facies on the forearc slope; (III) a calcareous ooze facies on isolated oceanic rises; (IV) a volcanic calcareous ooze facies on the broad, shallow arc platform; (V) a calcareous shell hash facies restricted to arc platform reefs; (VI) a calcareous volcanic silt facies with abundant pyroclastic turbidites in the backarc basin; (VII) a silty calcareous mud facies in backarc and forearc regions adjacent to continental sediment sources; and (VIII) a calcareous mud facies with terrigenous turbidites and contourites on the abyssal plain adjacent to continental sediment sources. The primary sources of sediment in the Lesser Antilles region include calcareous pelagic material, volcanogenic sediment from the island arc, and terrigenous sediment from South America. Factors governing the distribution of this sediment include: the level of the CCD, prevailing wind and ocean current patterns, bathymetry, distance from volcanogenic and terrigenous sediment sources, and fluctuations in sea level. A depositional model developed for the Lesser Antilles region serves as a type model for intraoceanic island arcs with large lateral terrigenous sediment influxes.
\end{abstract}

\section{INTRODUCTION}

Modern subduction zones are defined by deep, linear ocean trenches, with or without sediments, which can be studied only by geophysical profiling, bottom sediment sampling, or submersible observations. Such studies have been used to develop facies models for active margin trench and trench slope settings. These models are useful in identifying similar environments in ancient convergent margin sequences.

A number of localized studies have been completed that describe the sediment distribution and depositional processes operating at specific continental convergent margins (Middle America Trench: Ross, 1971; Heezen and Rawson, 1977; Underwood and Karig, 1980; von Huene, Aubouin, et al., 1980; and Moore, Watkins, et al., 1982. Eastern Aleutians: Piper et al., 1973. Oregon-Washington: Carlson and Nelson, 1969; Barnard, 1978; and Kulm and Scheidegger, 1979. Peru-Chile Trench: Scholl et al., 1970; Hayes, 1974; Krissek et al., 1980; Kulm et al., 1981; and Rosato and Kulm, 1981). Studies synthesizing lithofacies distributions and modeling general depositional processes at continental convergent margins are also available (Schweller and Kulm, 1978; Underwood and Bachman, 1982; and Underwood et al., 1980). These studies indicate that there is substantial variability in both the depositional geometries and the resultant sedimentary deposits found at these convergent margins.

Few studies of the sedimentary facies or depositional processes of intraoceanic convergent margins yet exist (Central Aleutians: Scholl et al., 1982. Marianas: Hussong, Uyeda, et al., 1982; and Lundberg, 1983). The Less-

\footnotetext{
${ }^{1}$ Biju-Duval, B., Moore, J. C., et al., Init. Repts. DSDP, 78A: Washington (U.S. Govt. Printing Office).

2 Present address: Ocean Drilling Program, Texas A\&M University, 500 University Drive, West, College Station, TX 77848.
}

er Antilles provides an example of an intraoceanic island arc in which there is a significant lateral source of terrigenous detritus. Abundant piston core, DSDP drill site, and seismic and bathymetric data allow very comprehensive analysis of the sediment distribution and depositional geometries in the Lesser Antilles (Fig. 1, back pocket). The piston cores detail modern areal sediment distributions and recent depositional processes, whereas the DSDP cores allow evaluation of temporal variations. Accordingly, I herein describe the sediment facies distribution and model the depositional processes of the Lesser Antilles.

\section{REGIONAL BACKGROUND}

\section{Physiographic and Tectonic Setting}

Late Cretaceous Atlantic oceanic crust currently is being underthrust beneath the Caribbean Plate at convergence rates of about $2 \mathrm{~cm} / \mathrm{y}$. along the Lesser Antilles arc (Fig. 2; Minster and Jordan, 1978). The volcanically active arc is $800 \mathrm{~km}$ long, extending from Saba in the north to Grenada in the south (Fig. 1). The southern part of the arc forms a narrow ridge that splits north of Dominica to form a broad platform. The Limestone Caribbee islands bounding the east side of this platform consist of Eocene-lower Miocene volcanic and intrusive rocks capped with Miocene limestones (Tomblin, 1975). Pliocene-Pleistocene volcanoes composing the Volcanic Caribbees, the currently active trace of island arc volcanism, occur along the west side of the platform (Martin-Kaye, 1969). The Limestone Caribbees probably represent an ancient trace of the Lesser Antilles volcanic arc.

The west side of the Lesser Antilles island arc is bounded by Grenada Basin, a backarc basin approximately $150 \mathrm{~km}$ wide with up to $7 \mathrm{~km}$ of sediment fill (Fig. 2; Biju-Duval et al., 1978). To the north the basin merges 

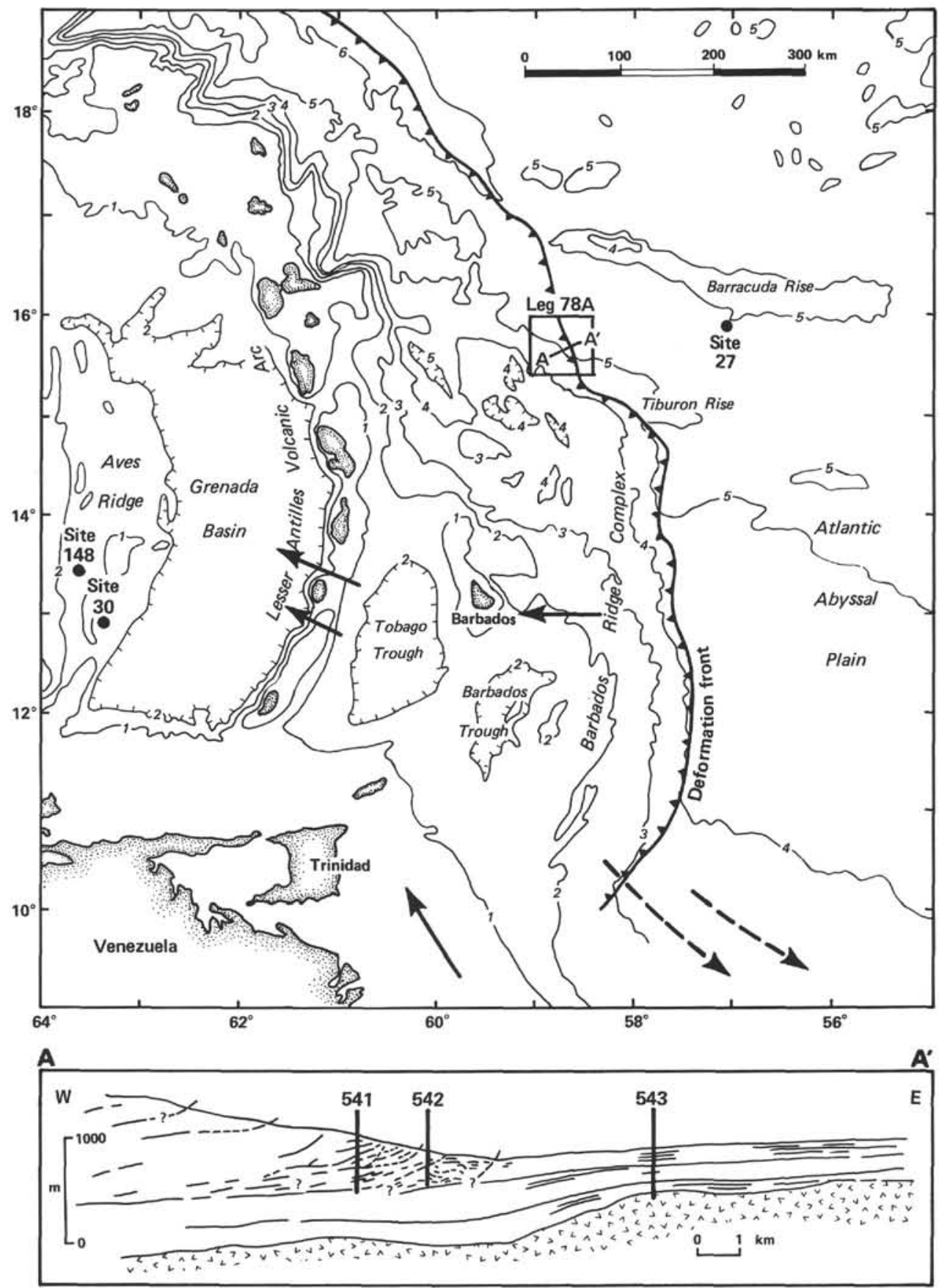

Figure 2. General location map of the Lesser Antilles intraoceanic island arc modified from Moore, BijuDuval, et al. (1982). (Bathymetric contours are in kilometers. Schematic cross section $\mathbf{A}-\mathbf{A}^{\prime}$ showing Leg 78A drill sites extends from the Atlantic abyssal plain into the deformed accretionary prism and was compiled from seismic lines A1C and A1D [see site reports, this volume]. Ocean surface currents are shown in solid arrows; bottom currents are shown in dashed arrows.)

with the volcanic arc and Aves Ridge to form a single plateau.

The forearc region east of the Lesser Antilles is approximately $450 \mathrm{~km}$ wide in the south, narrowing to less than $150 \mathrm{~km}$ in the north (Westbrook, 1975). The wide forearc region south of $15^{\circ} \mathrm{N}$ is underlain by a large accretionary prism constructed of sediment derived from nearby South American sources (Westbrook, 1982). The seaward boundary of the forearc region is marked by a folded and thrust-faulted deformation front (Marlow et al., 1974; Westbrook, 1975; Biju-Duval et al., 1978; Moore, Biju-Duval et al., 1982). A topographic trench marks the deformation front north of $16^{\circ} \mathrm{N}$.

The Barbados Ridge complex (Westbrook, 1982), a north-south trending pile of sediments up to $20 \mathrm{~km}$ thick near Barbados (Westbrook, 1975), is separated from the active arc by Tobago Trough forearc basin (Fig. 2). Tobago Trough contains more than $4 \mathrm{~km}$ of sediment fill 
that dips very gently toward the basin center and becomes progressively more deformed in the eastern portion of the basin (Keller et al., 1972; Mascle et al., 1979). These easternmost sediments are being overthrust by and tectonically incorporated into the Barbados Ridge complex (Biju-Duval et al., 1978).

Geologic mapping suggests that the Lesser Antilles has been active as an island arc at least since Eocene, and perhaps since Late Cretaceous (Tomblin, 1975; Lewis and Robinson, 1976; Westercamp, 1977; Biju-Duval et al, 1978). Aves Ridge, a largely submerged, inactive volcanic ridge located west of the modern island arc, is probably a remnant arc (Fig. 2; Fox et al., 1971; Nagle, 1972; Fox and Heezen, 1975; Biju-Duval et al., 1978; Nagle et al., 1978).

\section{Geologic History}

Our present understanding of the geologic history of the Lesser Antilles arc is based principally on onshore studies of the Lesser Antilles volcanic islands (R. C. Speed et al., "Lesser Antilles arc system and adjacent terranes"' unpublished final report to JOI, Inc., June 30, 1982: fig. 15) and Barbados (Speed, 1981; Pudsey and Reading, 1982), supplemented by dredge and core data where available. Sigurdsson et al. (1980) describe the two main types of volcanic products derived from eruptions along the arc: (1) principally silicic volcanics from the islands of Guadeloupe, Martinique, Domini$\mathrm{ca}$, and St. Lucia producing andesitic to rhyodacitic pumice fall deposits and ignimbrites; and (2) subordinate amounts of basic volcanics from the islands of St. Vincent and St. Lucia producing lava flows and airfall ash deposits of basaltic to basaltic-andesite composition.

The Upper Jurassic Basal Volcanic Complex (spilites and keratophyres) exposed on Désirade Island are the oldest rocks found in the Lesser Antilles; it is uncertain how they fit into the regional history of the area (Fink, 1972). The roughly north-south ridge represented by Grenada-Guadeloupe-Anguilla (Fig. 1) developed by the middle to late Eocene, as shown by the large amounts of volcanic rocks of that age exposed on those islands today (Christman, 1953; Martin-Kaye, 1959; Grunevald, 1965; Robinson and Jung, 1972). An early Miocene sub- mergence of the Lesser Antilles is documented by the growth of reefal limestone in many areas along the arc. Uplift and emergence of the arc ridge ended this period of Miocene limestone deposition and caused a shift in volcanic activity in the Lesser Antilles north of Dominica from the east to the west side of the broad platform (Tomblin, 1975). Volcanic activity has continued along that trace of the Lesser Antilles to the present day.

\section{Sediment Sources}

There are three major sources of sediment in the Lesser Antilles intraoceanic island arc region: (1) volcanogenic sediment in the form of pyroclastic debris flows or airfall ash layers derived from the volcanic island chain; (2) terrigenous material from South American river sources (Damuth, 1977); and (3) pelagic calcareous biogenic material. The sediment distribution observed in the Lesser Antilles intraoceanic island arc is controlled by factors such as the position of sea level, wind and ocean current patterns, bathymetry, location of the CCD, and distance from terrigenous and volcanogenic sediment supplies. The latter factor is particularly important, as the thickness of sediments found on the Atlantic abyssal plain varies from a maximum of about $4 \mathrm{~km}$ in the south near South America (Mascle et al., 1979) to about $700 \mathrm{~m}$ in DSDP Leg 78A drilling area (Moore, BijuDuval, et al., 1982).

\section{Stratigraphic-Sedimentologic Framework from DSDP Drill Sites}

In the Lesser Antilles region drilling has been done at a total of six DSDP sites (Table 1; Fig. 3, back pocket). Drilling results from DSDP Leg 78A (see site reports, this volume) provide information about the nature of the sedimentary section entering the subduction zone and the nature of the offscraped sedimentary section incorporated in the accretionary prism in the northern Lesser Antilles. Oceanic reference Site 543 recovered $409 \mathrm{~m}$ of pelagic and hemipelagic sediments overlying ocean crust (Fig. 3). The Holocene noncalcareous mud recovered at the top of Hole 543A (Core 543A-1) indicates that the seafloor at Site 543 is below the modern CCD. Sites 541 and 542 , both located near the toe of the accretionary

Table 1. Lesser Antilles core data used in this study.

\begin{tabular}{|c|c|c|c|}
\hline $\begin{array}{l}\text { Source of } \\
\text { sediment data }\end{array}$ & Type of data & Location of cores & Reference material \\
\hline $\begin{array}{l}\text { Lamont-Doherty } \\
\text { Geological Observatory } \\
\text { (LDGO) }\end{array}$ & $\begin{array}{l}92 \text { piston and gravity } \\
\text { cores }\end{array}$ & $\begin{array}{l}\text { Lesser Antilles backarc } \\
\text { and forearc regions } \\
\text { (Fig. 1) }\end{array}$ & $\begin{array}{l}\text { Damuth, } 1977 \\
\text { LDGO unpublished core descriptions } \\
\text { A. Wright (unpublished data) }\end{array}$ \\
\hline $\begin{array}{l}\text { Woods Hole } \\
\text { Oceanographic Institu- } \\
\text { tion } \\
\text { (WHOI) }\end{array}$ & $\begin{array}{l}30 \text { piston and gravity } \\
\text { cores }\end{array}$ & $\begin{array}{l}\text { Lesser Antilles backarc } \\
\text { and forearc regions } \\
\text { (Fig. 1) }\end{array}$ & $\begin{array}{l}\text { Johnson and Driscoll, } 1975 \\
\text { A. Wright (unpublished data) }\end{array}$ \\
\hline $\begin{array}{l}\text { University of Rhode } \\
\text { Island School of } \\
\text { Oceanography } \\
\text { (URI) }\end{array}$ & $\begin{array}{l}100 \text { piston and } \\
\text { gravity cores }\end{array}$ & $\begin{array}{l}\text { Lesser Antilles backarc } \\
\text { and forearc regions } \\
\text { (Fig. 1) }\end{array}$ & $\begin{array}{l}\text { Sigurdsson et al., } 1980 \\
\text { Williams, } 1977 \\
\text { URI unpublished core descriptions } \\
\text { A. Wright (unpublished data) }\end{array}$ \\
\hline Deep Sea Drilling Project & $\begin{array}{l}6 \text { rotary-cored } \\
\text { DSDP drill sites }\end{array}$ & $\begin{array}{l}\text { Sites } 27 \text { and 543: Atlantic } \\
\text { abyssal plain } \\
\text { Sites } 541 \text { and 542: toe of } \\
\text { accretionary prism in } \\
\text { northern Lesser } \\
\text { Antilles } \\
\text { Sites } 30 \text { and 148: Aves } \\
\text { Ridge (Fig. 1) }\end{array}$ & $\begin{array}{l}\text { Bader, Gerard, et al., } 1970 \mathrm{a} \\
\text { Edgar, Saunders, et al., } 1973 \mathrm{a} \\
\text { Sites } 541,542 \text {, and } 543 \text { reports, this } \\
\text { volume }\end{array}$ \\
\hline
\end{tabular}


prism, recovered Quaternary to Miocene hemipelagic sediments with interbedded volcanic ashes (Fig. 3). These sediments are lithologically and paleontologically similar to hemipelagic sediments of the same age recovered at Site 543, and are presumably offscraped ocean sediments (Moore, Biju-Duval, et al., 1982). The calcareous muds and marly calcareous oozes recovered at the top of Sites 541 and 542 indicate that the seafloor at those sites is above the modern CCD. No terrigenous turbidites were recovered at any of the Leg $78 \mathrm{~A}$ sites.

DSDP Site 27 sediments are dominantly hemipelagic and pelagic clays with several graded, quartz-rich terrigenous turbidite beds (Fig. 3; Bader et al., 1970b). The presence of these rare turbidite beds indicates that terrigenous sediments from South America are occasionally deposited as far north as the Barracuda Rise on the Atlantic abyssal plain.

DSDP Sites 30 and 148, both located on Aves Ridge, recovered calcareous silty clays and marly calcareous oozes interbedded with volcanic ash layers and volcanic sands (Fig. 3; Bader et al., 1970c; Edgar, Saunders, et al., 1973b). Calculated sediment accumulation rates, higher than those at Site 27, reflect the greater yield of volcanic material at these sites (see Wright, this volume).

\section{METHODS}

\section{Macroscopic and Microscopic Core Description}

In order to standardize the data base (see Table 1 for sources of data), I have visually redescribed all the piston cores recovered from the Lesser Antilles region (Fig. 1). However, due to drying and shrinkage of some of the core material, downcore colors and measured intervals reported for individual volcanic and terrigenous layers were taken verbatim from the available core descriptions.

Smear slides of the sediment at the top of each piston core were classified according to the standard DSDP sediment classification scheme (Supko et al., 1978). These data were used to construct a surface sediment lithofacies map (Fig. 4). Coarse-grained (dominantly silt-size) volcanogenic and terrigenous layers were described in detail both macroscopically and microscopically.

\section{Surface Sediment Carbonate Content}

To classify the surface sediment smear slides more accurately, the carbonate content of the surface sediment in each piston core was determined with a "Karbonate Bombe" (Müller and Gastner, 1971; Table 2; Fig. 5). Pure calcium carbonate standards were run every 20 samples. Carbonate determinations on duplicate samples were generally reproducible to within $2 \%$.

\section{Clay X-Ray Diffraction Analyses}

$\mathrm{X}$-ray diffraction analyses of the surface sediment clays were made on a selected subset of the piston cores to determine the modern clay mineral distribution in the Lesser Antilles arc. Additional clay results are published in Biscaye (1965), Griffin et al. (1968), and Griffin and Goldberg (1969); DSDP Leg 78A clay data are presented by Pudsey (this volume).

Samples for clay X-ray diffraction analysis were prepared and analyzed according to the procedure outlined by Hein et al. (1976). The $\mathrm{X}$-ray patterns were run on a Norelco X-ray diffractometer with nickel-filtered $\mathrm{CuK} \propto$ radiation and machine settings of $35 \mathrm{kV}$ and $20 \mathrm{~mA}$. Clay minerals were identified by their characteristic basal X-ray diffraction maxima, or "peaks". Clay percentages were determined on the glycolated X-ray analog records by the peak area method (Milliman and Bornhold, 1973), multiplied by Biscaye's (1965) weighting factors, and normalized to $100 \%$ (Table 3 ).

Table 3 lists both unweighted and weighted peak areas, for ease in later recalculation of the data to compare with other clay data generated with different weighting factors. The total area under the sum of the clay peak areas serves as an estimate of the quality of the percent- ages determined. Percentages were not calculated for profiles with very poorly defined peaks or very small peak areas. Reproducibility of results is $\pm 5 \%$.

\section{LITHOFACIES UNITS OF THE LESSER ANTILLES}

The lithofacies units discussed next are named both for their characteristic surface sediment lithologies and their depositional environments. The units are defined by their lithologies, macroscopic sedimentary structures, carbonate content, clay mineralogy, and calculated sediment accumulation rates. Table 4 summarizes the essential characteristics of each lithofacies unit, and Figure 4 shows the mapped distribution of these lithofacies units.

\section{(I) Pelagic Clay Facies: Deep Ocean Basin Environment}

This lithofacies unit is found on the Atlantic abyssal plain, below the CCD, and distant from South American terrigenous sediment sources (Fig. 4). The brown to dark yellowish brown bioturbated clays contain trace amounts of dispersed terrigenous silt, volcanic ash, and calcareous shell fragments; the percentages of these trace components are greatest along the deformation front

Table 2. Carbonate data from surface sediment piston-core samples.

\begin{tabular}{|c|c|c|c|c|c|c|c|}
\hline $\begin{array}{l}\text { Piston-core } \\
\text { sample }^{\mathrm{a}}\end{array}$ & $\underset{(\%)}{\mathrm{CaCO}_{3}}$ & $\begin{array}{l}\text { Piston-core } \\
\text { sample }^{\mathrm{a}}\end{array}$ & $\begin{array}{c}\mathrm{CaCO}_{3} \\
(\%)\end{array}$ & $\begin{array}{l}\text { Piston-core } \\
\text { sample }^{\mathrm{a}}\end{array}$ & $\underset{(\%)}{\mathrm{CaCO}_{3}}$ & $\begin{array}{l}\text { Piston-core } \\
\text { sample }^{\mathrm{a}}\end{array}$ & $\begin{array}{c}\mathrm{CaCO}_{3} \\
(\%)\end{array}$ \\
\hline GS-1 & 11 & $\mathrm{EN}-1$ & 84 & CHN36-7 & 55 & KM1-106 & 30 \\
\hline GS-2 & 12 & $\mathrm{EN}-2$ & 77 & CHN36-8 & 39 & KM1-107 & 32 \\
\hline GS-3 & 5 & EN-3 & 71 & CHN36-9 & 0 & KMI-108 & 21 \\
\hline GS -4 & 38 & EN-4 & 45 & CHN36-10 & 0 & KMI-109 & 19 \\
\hline GS-5 & 42 & EN-5 & 44 & CHN36-11 & 61 & KM1-110 & 37 \\
\hline GS-6 & 15 & EN-6 & 41 & CHN44-1 & 60 & KM1-112 & 21 \\
\hline GS-7 & 51 & EN-7 & 44 & CHN44-3 & 0 & KM1-113 & 18 \\
\hline GS-8 & 24 & EN-8 & 57 & CHN44-4 & 0 & KM1-114 & 21 \\
\hline GS-9 & 50 & EN-9 & 61 & CHN44-6 & 0 & KM1-115 & 24 \\
\hline GS-10 & 38 & EN-10 & 66 & CHN75-3 & 26 & KM1-116 & 54 \\
\hline GS-11 & 53 & EN-11 & 61 & CHN75-4 & 56 & KM1-118 & 48 \\
\hline GS- 12 & 48 & EN-12 & 61 & CHN75-5 & 56 & KM1-119 & 60 \\
\hline GS-13 & 35 & $\mathrm{EN}-13$ & 65 & CHN75- 6 & 6 & KM1-120 & 84 \\
\hline GS-14 & 32 & EN-14 & 68 & CHN75-7 & 6 & A $160-7$ & 0 \\
\hline GS-15 & 32 & $\mathrm{EN}-15$ & 76 & CHN75-30 & 16 & A160-8 & 0 \\
\hline GS-16 & 30 & EN-16 & 73 & CHN75-31 & 18 & A $160-9$ & 29 \\
\hline GS-17 & 16 & EN-18 & 67 & CHN75-32 & 61 & A181-7 & 17 \\
\hline GS-18 & 26 & EN-19 & 31 & CHN75-33 & 24 & A181-8 & 0 \\
\hline GS-21 & 0 & EN-22 & 67 & CHN75-34 & 0 & V12-91 & 30 \\
\hline GS- 22 & 0 & EN-23 & 63 & CHN75-35 & 0 & v12-92 & 29 \\
\hline GS-23 & 9 & EN-25 & 69 & CHN75-36 & 0 & V12-93 & 26 \\
\hline GS-24 & 8 & EN-26 & 0 & CHN75-37 & 0 & V15-175 & 33 \\
\hline GS-25 & 12 & EN-27 & 0 & All31-18 & 0 & v15-176 & 30 \\
\hline GS-26 & 11 & EN-28 & 55 & TR55-34 & 0 & V16-18 & 2 \\
\hline GS-27 & 16 & EN-29 & 51 & TR55-38 & 0 & V16-19 & 42 \\
\hline GS-28 & 18 & EN-30 & 60 & RC8-131 & 61 & V18-12 & 50 \\
\hline GS-29 & 0 & EN-31 & 30 & RC8-133 & 15 & V18-13 & 33 \\
\hline GS-31 & 0 & EN-32 & 9 & RC8-134 & 90 & V18-14 & 32 \\
\hline GS-39 & 13 & EN-33 & 9 & RC8.135 & 44 & v20-3 & 0 \\
\hline GS -40 & 10 & EN-34 & 5 & RC9-46 & 28 & $\mathrm{~V} 20-4$ & 71 \\
\hline GS -41 & 10 & EN-35 & 21 & RC9-47 & 42 & V20-5 & 50 \\
\hline GS-42 & 7 & EN-36 & 12 & RC9-50 & 8 & v20-6 & 16 \\
\hline GS-46 & 6 & EN-37 & 21 & RC13-171 & 13 & v20-7 & 15 \\
\hline GS-47 & 6 & EN-38 & 19 & RCl3-172 & 16 & v20-8 & 12 \\
\hline GS-48 & 6 & EN-39 & 25 & RCI3-173 & 6 & V $22-20$ & 0 \\
\hline GS-49 & 7 & EN-40 & 26 & RC13-174 & 6 & V23-127 & 1 \\
\hline GS-50 & 9 & EN-41 & 20 & RC13-175 & 30 & V23-128 & 0 \\
\hline GS-51 & 9 & $\mathrm{EN}-42$ & 21 & RCI3-176 & 17 & V23-129 & 41 \\
\hline GS-52 & 8 & $\mathrm{EN}-43$ & 17 & RCI5-179 & 26 & v23-130 & 9 \\
\hline GS. 53 & 28 & EN-44 & 13 & RC15-181 & 45 & $\mathrm{~V} 24-260$ & 53 \\
\hline GS-54 & 36 & EN-45 & 0 & RC16-33 & 2 & V25-38 & 21 \\
\hline GS-55 & 8 & EN-46 & 0 & RCI6-34 & 0 & V25-39 & 31 \\
\hline GS-56 & 22 & EN-47 & 15 & RCI6-35 & 0 & V26-111 & 0 \\
\hline GS-57 & 21 & EN-48 & 4 & RC16-36 & 0 & v26-112 & 7 \\
\hline GS-58 & 6 & EN-49 & 12 & RC16-37 & 0 & $\mathrm{v} 26-113$ & 22 \\
\hline GS-59 & 30 & EN-50 & 16 & RC16-38 & 0 & V26-114 & 30 \\
\hline GS -60 & 11 & EN-51 & 13 & RC16-39 & 11 & V26-115 & 48 \\
\hline GS-61 & 10 & EN-54 & 9 & $\mathrm{RC} 16-40$ & 43 & V30-14 & 32 \\
\hline GS-62 & 7 & EN-55 & 7 & RC16-41 & 45 & V30-15 & 0 \\
\hline GS -63 & 0 & CHN36-3 & 0 & RC16-42 & 48 & v31-143 & 26 \\
\hline GS-64 & 0 & CHN36-4 & 0 & RC16-43 & 35 & V31-147 & 51 \\
\hline GS-65 & 0 & CHN36-5 & 0 & KM1-104 & so & V $31-148$ & 28 \\
\hline \multirow[t]{2}{*}{ GS-66 } & 44 & CHN36-6 & 0 & KM1-105 & 36 & V31-149 & 8 \\
\hline & & & & & & v31-150 & 0 \\
\hline
\end{tabular}




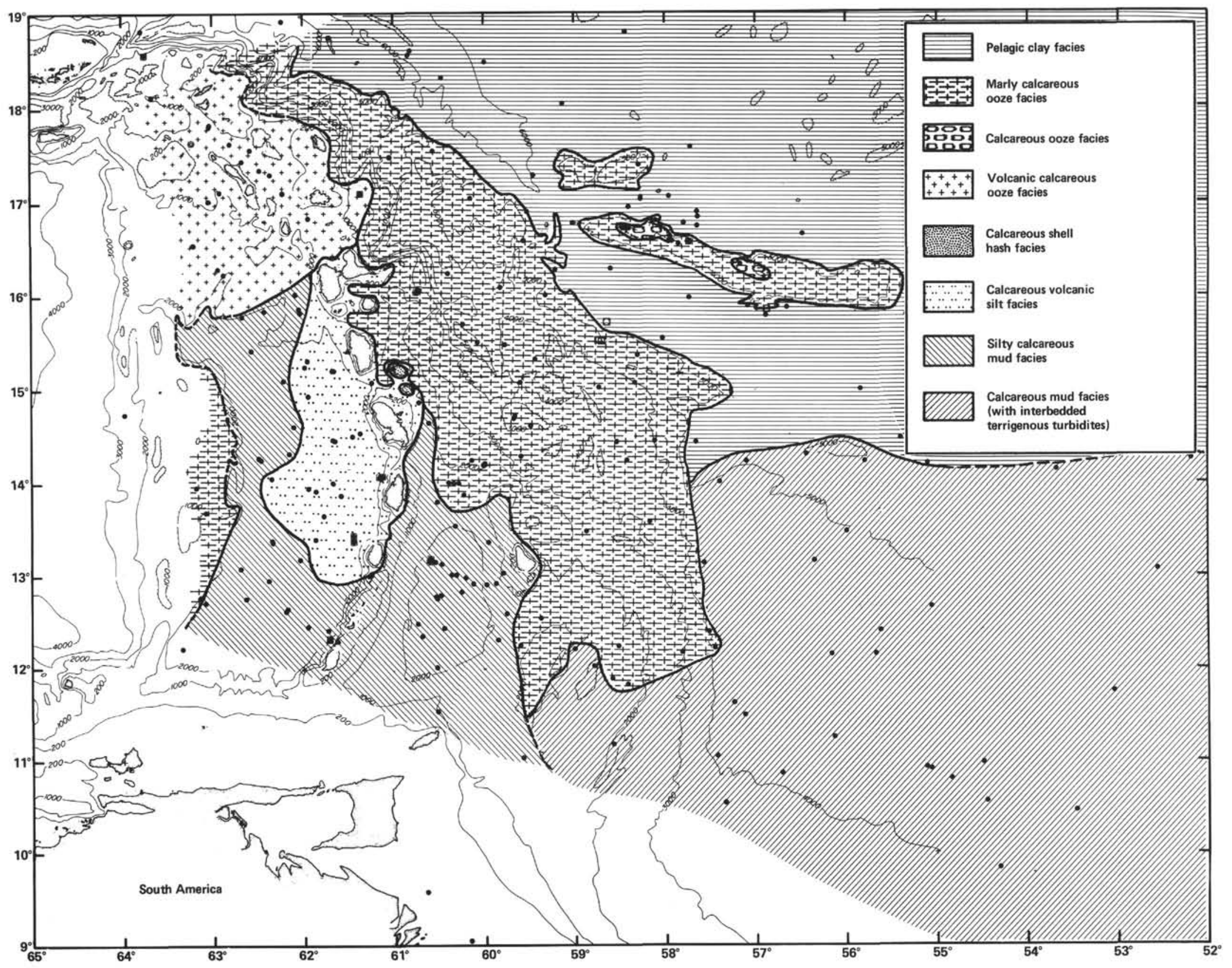

Figure 4. Map of lithofacies unit distribution for the Lesser Antilles intraoceanic island arc region. 


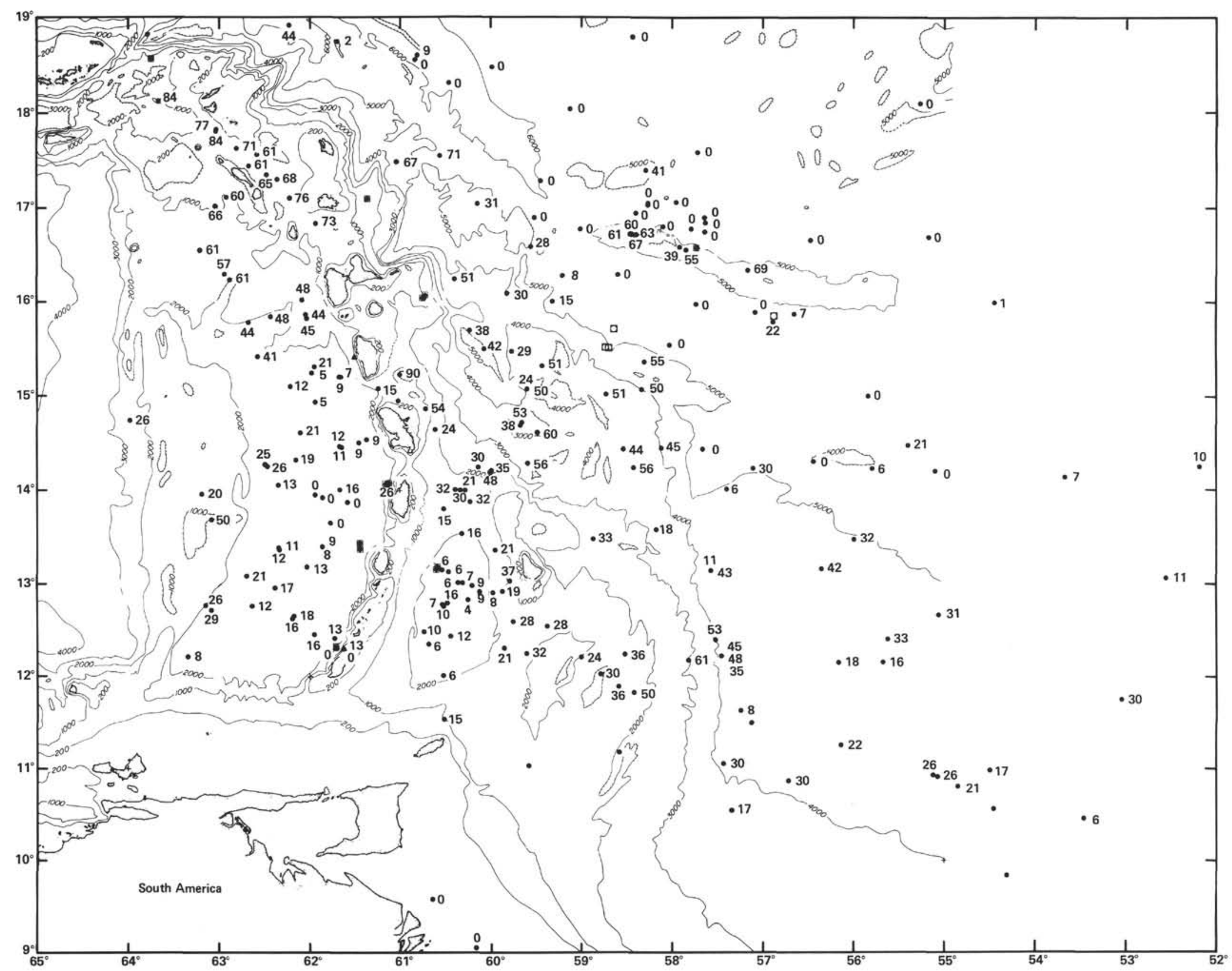

Figure 5. Calcium carbonate percentage in surface sediments from piston cores in the Lesser Antilles intraoceanic island arc region. (Calcium carbonate percentages were determined with the "Karbonate Bombe" [Müller and Gastner, 1971]. Calcium carbonate data are listed in Table 2.) 
Table 3. Clay mineralogy data from surface sediment piston-core samples.

\begin{tabular}{|c|c|c|c|c|c|c|c|c|c|c|c|c|c|c|}
\hline \multirow{2}{*}{$\begin{array}{c}\text { Piston-core } \\
\text { sample } \\
\text { (interval in } \mathrm{cm} \text { ) }\end{array}$} & \multicolumn{3}{|c|}{$\begin{array}{l}\text { Unweighted } \\
\text { peak area }\end{array}$} & \multicolumn{3}{|c|}{$\begin{array}{l}\text { Weighted } \\
\text { peak area }\end{array}$} & \multirow{2}{*}{$\begin{array}{c}\text { Total } \\
\text { weighted } \\
\text { peak area }\end{array}$} & \multicolumn{3}{|c|}{$\begin{array}{c}\text { Clay } \\
\text { mineral } \\
\text { percentage }\end{array}$} & \multicolumn{2}{|c|}{$\begin{array}{c}\text { Peak } \\
\text { height }\end{array}$} & \multicolumn{2}{|c|}{$\begin{array}{c}\text { Clay } \\
\text { mineral } \\
\text { percentage }\end{array}$} \\
\hline & $\mathrm{K}+\mathrm{C}$ & I & M & $\mathrm{K}+\mathrm{C}$ & I & M & & $\mathrm{K}+\mathrm{C}$ & I & M & K & C & K & C \\
\hline GS-2, 0-2 & 11.8 & 5.0 & 8.5 & 23.5 & 19.8 & 8.5 & 51.8 & 45 & 38 & 17 & 9.7 & 5.2 & 29 & 16 \\
\hline GS-3, 0-2 & 7.2 & 1.4 & 7.9 & 14.4 & 8.9 & 7.9 & 31.2 & 46 & 28 & 25 & 6.0 & 3.0 & 31 & 15 \\
\hline GS-4, 0-2 & 19.6 & 7.6 & 10.6 & 39.1 & 30.3 & 10.6 & 80.0 & 49 & 38 & 13 & 15.1 & 10.1 & 29 & 20 \\
\hline GS-5, 0-2 & 22.2 & 7.4 & 9.5 & 44.4 & 29.8 & 9.5 & 83.7 & 53 & 36 & 11 & 15.8 & 10.4 & 32 & 21 \\
\hline GS $-7,0-2$ & 25.6 & 8.4 & 13.1 & 51.3 & 33.3 & 13.1 & 97.7 & 53 & 34 & 13 & 18.4 & 9.3 & 35 & 18 \\
\hline GS-21, 0-2 & 8.2 & 2.5 & 10.2 & 16.4 & 10.0 & 10.2 & 28.0 & 45 & 27 & 28 & 7.4 & 3.8 & 30 & 15 \\
\hline GS-22, 0-2 & 9.6 & 3.4 & 9.8 & 19.1 & 13.4 & 9.8 & 42.3 & 45 & 32 & 23 & 7.8 & 3.7 & 31 & 14 \\
\hline GS-25, 0-2 & 28.4 & 10.8 & 20.6 & 56.9 & 43.2 & 20.6 & 120.7 & 47 & 36 & 17 & ? & $?$ & $?$ & $?$ \\
\hline GS- $26,0-2$ & 17.7 & 5.3 & 8.3 & 35.4 & 21.3 & 8.3 & 65.0 & 54 & 34 & 13 & 14.0 & 6.6 & 37 & 17 \\
\hline GS-27, 0-2 & 29.4 & 11.9 & 14.3 & 58.7 & 47.6 & 14.3 & 120.6 & 49 & 39 & 12 & $?$ & $?$ & $?$ & $?$ \\
\hline GS- $28,0-2$ & 24.8 & 9.4 & 13.8 & 49.6 & 37.4 & 13.8 & 100.8 & 49 & 37 & 14 & $?$ & $?$ & $?$ & $?$ \\
\hline GS $-40,0-2$ & 17.4 & 5.8 & 8.5 & 34.8 & 23.1 & 8.5 & 66.4 & 52 & 35 & 13 & 14.5 & 7.5 & 34 & 18 \\
\hline GS- $53,0-2$ & 17.6 & 8.9 & 6.6 & 35.3 & 35.6 & 6.6 & 77.5 & 46 & 46 & 8 & 13.3 & 7.1 & 30 & 16 \\
\hline GS-54, $0-2$ & 16.9 & 6.3 & 6.4 & 33.8 & 25.3 & 6.4 & 65.5 & 52 & 39 & 9 & 12.9 & 6.9 & 34 & 18 \\
\hline GS- $55,0-2$ & 13.3 & 5.8 & 6.0 & 26.6 & 23.4 & 6.0 & 56.0 & 47 & 42 & 11 & 10.1 & 6.3 & 29 & 18 \\
\hline GS- $57,0-2$ & 15.2 & 6.6 & 7.8 & 30.3 & 26.2 & 7.8 & 64.3 & 47 & 41 & 12 & 10.6 & 5.2 & 31 & 16 \\
\hline GS- $58,0-2$ & 14.1 & 6.6 & 6.6 & 28.1 & 26.6 & 6.6 & 61.3 & 46 & 43 & 11 & 9.5 & 4.9 & 30 & 13 \\
\hline GS- $59,0-2$ & 14.8 & 5.4 & 5.9 & 29.5 & 21.6 & 5.9 & 57.0 & 52 & 38 & 10 & 10.2 & 5.4 & 34 & 18 \\
\hline GS- $60,0-2$ & 19.1 & 8.7 & 9.1 & 38.2 & 34.8 & 9.1 & 82.1 & 47 & 42 & 11 & 14.0 & 8.2 & 30 & 17 \\
\hline GS-61, 0-2 & 13.6 & 6.7 & 9.1 & 27.1 & 26.9 & 9.1 & 63.1 & 43 & 43 & 14 & 9.7 & 5.5 & 28 & 15 \\
\hline GS- $62,0-2$ & 15.8 & 5.8 & 6.9 & 31.6 & 23.1 & 6.9 & 61.6 & 51 & 38 & 11 & 12.1 & 6.5 & 33 & 18 \\
\hline GS- $63,0-2$ & 15.0 & 6.6 & 6.3 & 30.1 & 26.6 & 6.3 & 63.0 & 48 & 42 & 10 & 11.8 & 6.2 & 32 & 16 \\
\hline GS- $64,0-2$ & 24.2 & 10.6 & 7.9 & 48.3 & 42.6 & 7.9 & 98.8 & 49 & 43 & 8 & 16.7 & 9.4 & 31 & 18 \\
\hline GS-65, 0-2 & 18.7 & 8.4 & 6.8 & 37.4 & 33.5 & 6.8 & 77.7 & 48 & 43 & 9 & 13.3 & 8.4 & 29 & 19 \\
\hline GS-66, 0-2 & 9.2 & 4.1 & 3.6 & 18.3 & 16.5 & 3.6 & 38.4 & 48 & 43 & 9 & 7.6 & 4.6 & 30 & 18 \\
\hline EN-4, 0-2 & 7 & 2.6 & 5.1 & 15.4 & 10.5 & 5.1 & 31.0 & 50 & 34 & 16 & 6.6 & 2.3 & 37 & 13 \\
\hline EN-5, 0-2 & 7.3 & 2.8 & 4.3 & 14.6 & 11.2 & 4.3 & 30.1 & 49 & 37 & 1. & 7.0 & 3. & 32 & 15 \\
\hline EN-6, 0-2 & 10.8 & 2.9 & 6.6 & 21.7 & 11.7 & 6.6 & 40.0 & 54 & 29 & 17 & 9.6 & 4. & 37 & 17 \\
\hline EN-7, 0-2 & 9.8 & 3.4 & 6.7 & 19.7 & 13.5 & 6.7 & 39.9 & 49 & 34 & 17 & 9.2 & 4. & 32 & 17 \\
\hline EN-8, 0-2 & 9.6 & 2.6 & 6.1 & 19.1 & 10.1 & 6.1 & 35.3 & 54 & 29 & 17 & 10.4 & 4.9 & 37 & 17 \\
\hline EN-9, 0-2 & 7.4 & 2.7 & 4.5 & 14.8 & 10.9 & 4.5 & 30.2 & 49 & 36 & 15 & 6.5 & 3.1 & 33 & 16 \\
\hline $\mathrm{EN}-10,0-2$ & 8.9 & 3.7 & 6.7 & 17.8 & 14.9 & 6.7 & 39.4 & 45 & 38 & 17 & 10.9 & 5.4 & 30 & 15 \\
\hline $\mathrm{EN}-11,0-2$ & 3.0 & 0.8 & 4.2 & 6.1 & 3.2 & 4.2 & 13.4 & 45 & 24 & 31 & 2.7 & 1. & 27 & 18 \\
\hline $\mathrm{EN}-18,0-2$ & 15.2 & 3.9 & 8.7 & 30.4 & 15.7 & 8.7 & 54.8 & 55 & 29 & 16 & 9.7 & 9.6 & 28 & 27 \\
\hline $\mathrm{EN}-19,0-2$ & 16.0 & 6.4 & 7.1 & 32.1 & 25.5 & 7.1 & 64.7 & 50 & 39 & 11 & 11.2 & 10.8 & 26 & 24 \\
\hline $\mathrm{EN}-22,0-2$ & 12.9 & 5.6 & 5.3 & 25.8 & 22.4 & 5.3 & 53.5 & 48 & 42 & 10 & 11.5 & 6.5 & 31 & 17 \\
\hline EN-23, 0-2 & 22.0 & 8.0 & 8.2 & 44.1 & 32.2 & 8.2 & 84.5 & 52 & 38 & 10 & 18.8 & 10.9 & 33 & 19 \\
\hline EN-25, 0-2 & 24.5 & 11.6 & 15.7 & 49.0 & 46.2 & 15.7 & 110.9 & 44 & 42 & 14 & 14.9 & 6.9 & 30 & 14 \\
\hline EN-26, 0-2 & 20.8 & 8.8 & 10.9 & 41.5 & 35.4 & 10.9 & 87.8 & 47 & 40 & 13 & 16.7 & 10.1 & 29 & 18 \\
\hline EN-27, 0-2 & 30.4 & 13.8 & 15.5 & 60.7 & 55.4 & 15.5 & 131.6 & 46 & 42 & 12 & $?$ & $?$ & $?$ & $?$ \\
\hline EN-28, 0-2 & 21.6 & 9.2 & 11.2 & 43.2 & 36.7 & 11.2 & 91.1 & 47 & 40 & 13 & 12.1 & 8.2 & 28 & 19 \\
\hline EN-29, 0-2 & 13.1 & 4.2 & 6.3 & 26.2 & 17.0 & 6.3 & 49.5 & 53 & 34 & 13 & 11.4 & 6. & 34 & 19 \\
\hline EN-30, 0-2 & 7.6 & 2.6 & 4.4 & 15.2 & 10.6 & 4.4 & 30.2 & 50 & 35 & 15 & 6.8 & 4. & 32 & 18 \\
\hline EN-31, 0-2 & 6.4 & 2.6 & 3.2 & 12.8 & 10.2 & 3.2 & 26.2 & 49 & 39 & 12 & 5.6 & 3. & 31 & 18 \\
\hline EN-32, 0-2 & 5.0 & 2.6 & 2.2 & 10.1 & 10.5 & 2. & 22.8 & 44 & 46 & 10 & 3.7 & 2. & 26 & 18 \\
\hline EN-33, 0-2 & 3.2 & 1.2 & 2.7 & 6.4 & 4.6 & 2.7 & 13.7 & 47 & 33 & 20 & 3.4 & 2.4 & 28 & 19 \\
\hline EN-34, 0-2 & 2.9 & 0.9 & 4.6 & 5.8 & 3.7 & 4.6 & 14.1 & 41 & 26 & 33 & $?$ & $?$ & $?$ & $?$ \\
\hline EN-35, 0-2 & 5.6 & 0.4 & 2.6 & 11.3 & 1.5 & 2.6 & 15.4 & 74 & 9 & 17 & 4.4 & 2.3 & 49 & 25 \\
\hline EN-48, 0-2 & 12.0 & 4.7 & 5.0 & 24.0 & 18.8 & 2.6 & 47.8 & so & 39 & 11 & 9.0 & 4.8 & 32 & 18 \\
\hline $\mathrm{CHN}$ & 24.9 & 11.2 & 9. & 49.8 & 45 & 9.4 & 104.2 & 4 & 43 & 9 & 16 & 9. & 30 & 18 \\
\hline CHN36-10, 0-2 & 25.8 & 11.0 & 11.6 & & 43.9 & 11.6 & 107.2 & 40 & 41 & 11 & 17.2 & 10.2 & 30 & 18 \\
\hline CHN75-37, 0-2 & 11.3 & 6.6 & 3.7 & 22.6 & 26.2 & 3.7 & 52.5 & 43 & 50 & 7 & 7.7 & 5.6 & 25 & 18 \\
\hline $\mathrm{RCl}$ & 15 & 8. & 8. & & 34. & 8. & 81 & 47 & 42 & 11 & 13.6 & 7. & 31 & 16 \\
\hline $\mathbf{R C}$ & & 7. & 8 & & 28 & 8 & 70 & 48 & 40 & 12 & 11.8 & s. & 32 & 16 \\
\hline RC16-40, 0-2 & 23.9 & 14.2 & 8.7 & 47. & 56.8 & 8.7 & 113.3 & 42 & 50 & 8 & 16.5 & 10.2 & 26 & 16 \\
\hline $\mathrm{RC} 16-44,0-2$ & 18.6 & 6.8 & 10.1 & 37.2 & 27.0 & 10.1 & 74.3 & 50 & 36 & 14 & 14.2 & 7.1 & 34 & 16 \\
\hline V20-3 & . & 7.2 & 7. & & 28. & 7. & 66 & 4 & 43 & 11 & 10 & & 30 & 16 \\
\hline V $25-39,0-2$ & 18. & 9.2 & 6.9 & & 36.6 & 6.9 & & 40 & 45 & 9 & 11.3 & 7.0 & 28 & 18 \\
\hline$v 31-150,0-2$ & 23.3 & 9.9 & 12.2 & 46.6 & 39.6 & 12.2 & 98.4 & 47 & 40 & 13 & 19.4 & 10.3 & 30 & 17 \\
\hline TR55 & 14. & 7.4 & 5. & 29 & 29. & 5. & 64 & 45 & 46 & 9 & 11.2 & 6. & 29 & 16 \\
\hline TR55-38, $0-2$ & 23.0 & 9.4 & 8.7 & 46.0 & 37.4 & 8.7 & 92.1 & 50 & 41 & 9 & 14.2 & 6.7 & 34 & 16 \\
\hline
\end{tabular}

Note: $\mathrm{K}=$ kaolinite; $\mathrm{C}=$ chlorite; $\mathrm{M}=$ montmorillonite; $\mathrm{I}=$ illite. See Table 2 for cruise identifiers.

and immediately north and south of the Barracuda Rise. This fact is presumably the result of ocean current transport of South American terrigenous detritus north along the deformation front, eolian transport of volcanogenic detritus eastward (Sigurdsson et al., 1980), and downslope movement of biogenous material from the Barracuda Rise and Barbados Ridge, respectively. Sediment accumulation rates for this facies range from 0.8 to $1.7 \mathrm{~g} /\left(\mathrm{cm}^{2} \cdot 10^{3} \mathrm{y}\right.$. $)$; these values are typical for oceanic pelagic sediments (see Wright, this volume; Worsley and Davies, 1979; Sigurdsson et al., 1980; Shephard and McMillen, 1982).

White to light gray, moderately to well-sorted ash layers compose up to $10 \%$ of the cored material, with ash content decreasing to the east (Fig. 3). These layers are between 1 and $15 \mathrm{~cm}$ thick and are composed of abun- dant glass shards with varying amounts of crystals, clay, and carbonate; they show sharp bases and either sharp or bioturbated tops (Fig. 6; Sigurdsson et al., 1980). Many ash layers contain basal crystal-rich laminae, resulting from differential gravitational settling through the water column. Notably, montmorillonite percentages also decrease eastward with increasing distance from island arc volcanic sources (Fig. 7).

Several piston cores from the pelagic clay facies contain calcareous (biogenic) or terrigenous turbidites. Piston Cores RC8-135, RC16-33, and RC16-34 (Fig. 1) contain graded, laminated, 0.1 to $1.0 \mathrm{~m}$ thick, biogenic turbidites with sharp bases and bioturbated tops. These turbidites are derived from the adjacent shallow arc platform and transported down local canyons in the continental slope to deep ocean basin depositional sites 
Table 4. Summary characteristics of Lesser Antilles lithofacies units.

\begin{tabular}{|c|c|c|c|c|c|c|}
\hline & $\begin{array}{l}\text { Depositional } \\
\text { environment }\end{array}$ & $\begin{array}{l}\text { Composition } \\
\text { of surface } \\
\text { sediment }\end{array}$ & $\begin{array}{c}\mathrm{CaCO}_{3} \\
(\%)\end{array}$ & $\begin{array}{l}\text { Clay } \\
\text { mineralogy } \\
(\%)\end{array}$ & Cored sediments & $\begin{array}{l}\text { Sediment } \\
\text { accumulation } \\
\text { rates }\end{array}$ \\
\hline Pelagic clay facies & $\begin{array}{l}\text { Abyssal plain below } \\
\text { CCD and dis- } \\
\text { tant from } \\
\text { terrigenous } \\
\text { sediment source }\end{array}$ & $\begin{array}{l}\text { Pelagic clay with small } \\
\text { percent dispersed ash } \\
\text { and terrigenous silt and } \\
\text { shell fragments }\end{array}$ & $0-10$ & $\begin{aligned} \mathrm{I} & =38-43 \\
\mathrm{~K} & =29-32 \\
\mathrm{M} & =7-13 \\
\mathrm{C} & =16-19\end{aligned}$ & $\begin{array}{l}\text { Clay interbedded with } \\
\text { ash layers and } \\
\text { rare thin biogenic } \\
\text { turbidites and } \\
\text { terrigenous silts }\end{array}$ & $0.8-1.7 \mathrm{~g} /\left(\mathrm{cm}^{2} \cdot 10^{3} \mathrm{y}.\right)$ \\
\hline $\begin{array}{l}\text { Marly calcareous } \\
\text { ooze facies }\end{array}$ & $\begin{array}{l}\text { Ocean ridges; slope } \\
\text { environment } \\
\text { above } C C D\end{array}$ & $\begin{array}{l}30-60 \% \text { calcareous micro- } \\
\text { fossils; }<30 \% \text { dis- } \\
\text { persed ash and terrige- } \\
\text { nous silt; clay }\end{array}$ & $30-60$ & $\begin{aligned} \mathrm{I} & =34-46 \\
\mathrm{~K} & =26-35 \\
\mathrm{M} & =8-15 \\
\mathrm{C} & =16-24\end{aligned}$ & $\begin{array}{l}\text { Marly calcareous } \\
\text { ooze interbedded } \\
\text { with ash layers } \\
\text { and local biogenic } \\
\text { sands }\end{array}$ & $3.3-5.8 \mathrm{~g} /\left(\mathrm{cm}^{2} \cdot 10^{3} \mathrm{y}.\right)$ \\
\hline $\begin{array}{l}\text { Calcareous ooze } \\
\text { facies }\end{array}$ & $\begin{array}{l}\text { Ocean ridges above } \\
\text { CCD }\end{array}$ & $\begin{array}{l}\text { 60-100\% calcareous mi- } \\
\text { crofossils; few percent } \\
\text { dispersed ash and trace } \\
\text { terrigenous silt }\end{array}$ & $60-100$ & $\begin{aligned} \mathrm{I} & =38-42 \\
\mathrm{~K} & =30-33 \\
\mathrm{M} & =10-14 \\
\mathrm{C} & =14-20\end{aligned}$ & $\begin{array}{l}\text { Calcareous ooze } \\
\text { interbedded with } \\
\text { ash layers; no } \\
\text { terrigenous or } \\
\text { biogenic turbidites }\end{array}$ & $1.7-3.3 \mathrm{~g} /\left(\mathrm{cm}^{2} \cdot 10^{3} \mathrm{y}.\right)$ \\
\hline $\begin{array}{l}\text { Volcanic calcareous } \\
\text { ooze facies }\end{array}$ & $\begin{array}{l}\text { Broad, shallow arc } \\
\text { platform }\end{array}$ & $\begin{array}{l}60-100 \% \text { calcareous mi- } \\
\text { crofossils; } 0-40 \% \\
\text { volcanic silt }\end{array}$ & $60-100$ & $\begin{aligned} \mathrm{I} & =29-38 \\
\mathrm{~K} & =28-33 \\
\mathrm{M} & =13-19 \\
\mathrm{C} & =15-18\end{aligned}$ & $\begin{array}{l}\text { Volcanic calcareous } \\
\text { ooze interbedded } \\
\text { with volcanic } \\
\text { calcareous (bio- } \\
\text { genic) turbidites } \\
\text { and ash layers }\end{array}$ & \\
\hline $\begin{array}{l}\text { Calcareous shell } \\
\text { hash facies }\end{array}$ & $\begin{array}{l}\text { Local reefs on arc } \\
\text { platform }\end{array}$ & $\begin{array}{l}100 \% \text { shell and coral } \\
\text { fragments }\end{array}$ & about $100 \%$ & & $\begin{array}{l}\text { Whole core consists } \\
\text { of shell and coral }\end{array}$ & \\
\hline $\begin{array}{l}\text { Calcareous vol- } \\
\text { canic silt facies }\end{array}$ & $\begin{array}{l}\text { Backarc basin close } \\
\text { to volcanic } \\
\text { island chain }\end{array}$ & $\begin{array}{l}70-90 \% \text { volcanic sand and } \\
\text { silt; } 10-30 \% \text { calcareous } \\
\text { microfossils; minor clay }\end{array}$ & $10-30$ & $\begin{aligned} \mathrm{I} & =26-38 \\
\mathrm{~K} & =26-31 \\
\mathrm{M} & =17-31 \\
\mathrm{C} & =11-15\end{aligned}$ & $\begin{array}{l}\text { Cores contain many } \\
\text { volcanic sands } \\
\text { and pyroclastic } \\
\text { turbidites }\end{array}$ & $10.8-15.8 \mathrm{~g} /\left(\mathrm{cm}^{2} \cdot 10^{3}\right.$ y. $)$ \\
\hline $\begin{array}{c}\text { Silty calcareous } \\
\text { mud facies }\end{array}$ & $\begin{array}{l}\text { Backarc and fore- } \\
\text { arc regions } \\
\text { close to conti- } \\
\text { nental sediment } \\
\text { source }\end{array}$ & $\begin{array}{l}10-30 \% \text { calcareous micro- } \\
\text { fossils; } 10-30 \% \text { dis- } \\
\text { persed volcanic terrige- } \\
\text { nous silt; }>40 \% \text { clay }\end{array}$ & $10-30$ & $\begin{aligned} \mathrm{I} & =26-43 \\
\mathrm{~K} & =31-37 \\
\mathrm{M} & =9-18 \\
\mathrm{C} & =15-18\end{aligned}$ & $\begin{array}{l}\text { Cores contain local } \\
\text { pyroclastic turbi- } \\
\text { dites, biogenic } \\
\text { and volcanic } \\
\text { sands, and ash } \\
\text { layers }\end{array}$ & \\
\hline $\begin{array}{l}\text { Calcareous mud } \\
\text { facies }\end{array}$ & $\begin{array}{l}\text { Abyssal plain above } \\
\text { CCD close to } \\
\text { continental } \\
\text { sediment source }\end{array}$ & $\begin{array}{l}10-30 \% \text { calcareous micro- } \\
\text { fossils; }<10-25 \% \\
\text { dispersed terrigenous } \\
\text { silt; }>60 \% \text { clay }\end{array}$ & $10-30$ & $\begin{aligned} \mathrm{I} & =38-50 \\
\mathrm{~K} & =26-34 \\
\mathrm{M} & =8-14 \\
\mathrm{C} & =15-18\end{aligned}$ & $\begin{array}{l}\text { Calcareous mud } \\
\text { interbedded with } \\
\text { thin ash layers } \\
\text { and terrigenous } \\
\text { turbidites and } \\
\text { contourites }\end{array}$ & \\
\hline
\end{tabular}

${ }^{a_{I}}=$ illite; $\mathrm{K}=$ kaolinite; $\mathrm{M}=$ montmorillonite; $\mathrm{C}=$ chlorite. See Wright, this volume.

(Fig. 3; Fig. 8). Piston Core V26-111 (Fig. 1) contains two organic-rich clay layers approximately $0.1 \mathrm{~m}$ thick probably derived from the South American continent and transported north along the deformation front by turbidity currents (Fig. 3). DSDP Site 27 recovered several terrigenous turbidites derived from South America; a distance of about $850 \mathrm{~km}$ transport is indicated (Fig. 3; Bader et al., 1970b). Site 543, located a comparable distance from the South American continent, does not contain any terrigenous turbidites because of its isolation on the Tiburon Rise (Moore, Biju-Duval, et al., 1982).

\section{(II) Marly Calcareous Ooze Facies: Slope Environment}

Bioturbated yellowish brown marly calcareous oozes are found at water depths above the CCD on the Barracuda Rise, the Aves Ridge, and the accretionary prism north of $12^{\circ} \mathrm{N}$ latitude (Fig. 4). Piston cores collected from this facies contain volcanic ash layers similar to those described for the pelagic clay facies. The thickest and most abundant ash layers and the highest montmorillonite contents are found in cores taken immediately east of the islands of Dominica, Martinique, St. Lucia,
Guadeloupe, and St. Vincent where most of the volcanic activity is concentrated (Figs. 3 and 7; Sigurdsson et al., 1980). Sediment accumulation rates range from 3.3 to $5.8 \mathrm{~g} /\left(\mathrm{cm}^{2} \cdot 10^{3} \mathrm{y}\right.$.), significantly higher than rates reported for oceanic pelagic sediments (see Wright, this volume; Damuth, 1977).

Graded calcareous sand layers up to $55 \mathrm{~cm}$ thick were recovered in piston cores located on the east side of Aves Ridge (V12-93, EN-41), in small topographic depressions on the accretionary prism (GS-4, GS-5), and close to the arc platform (V31-147) (Fig. 3). In addition, Piston Core EN-30 contains several thin volcanic sand layers (Fig. 3). These calcareous and volcanic sand layers result from downslope movement of material from the adjacent shallow arc platform. Significantly, there are no terrigenous silt or sand layers found in any of the slope facies cores, indicating that terrigenous material from South America is not deposited directly on the trench slope.

\section{(III) Calcareous Ooze Facies: Ocean Ridges and Rises}

Pale brown, bioturbated calcareous oozes are restricted to the shallowest portions of the Barracuda Rise, above the CCD, and distant from terrigenous sediment sources (Fig. 4). These oozes are characterized by greater than 


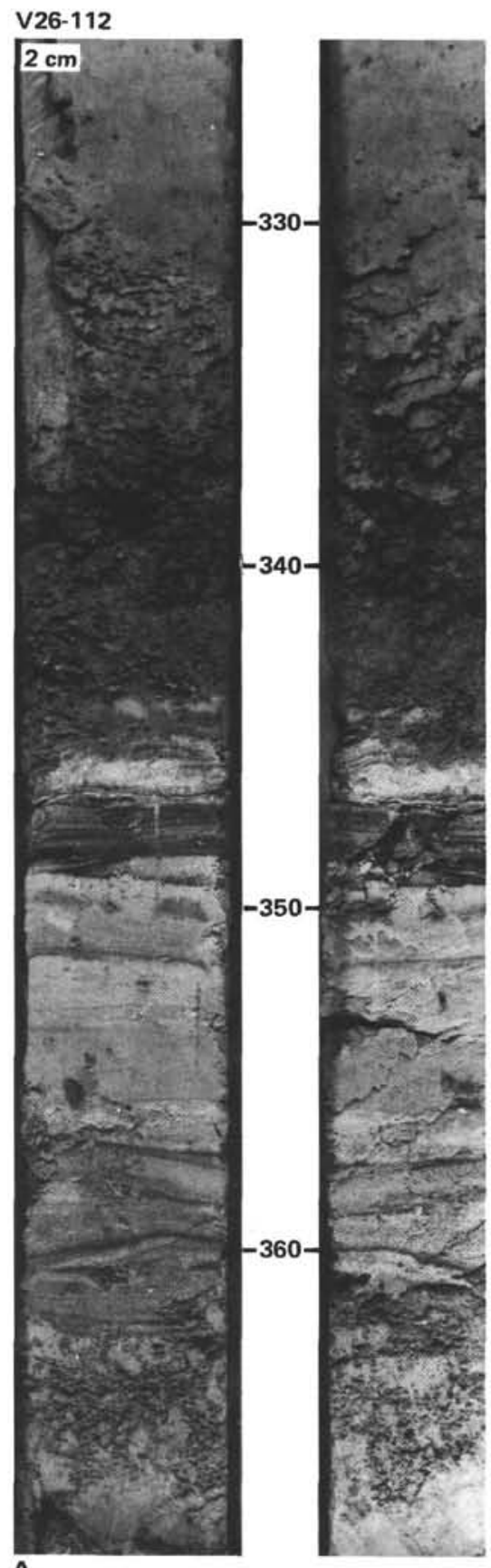

A

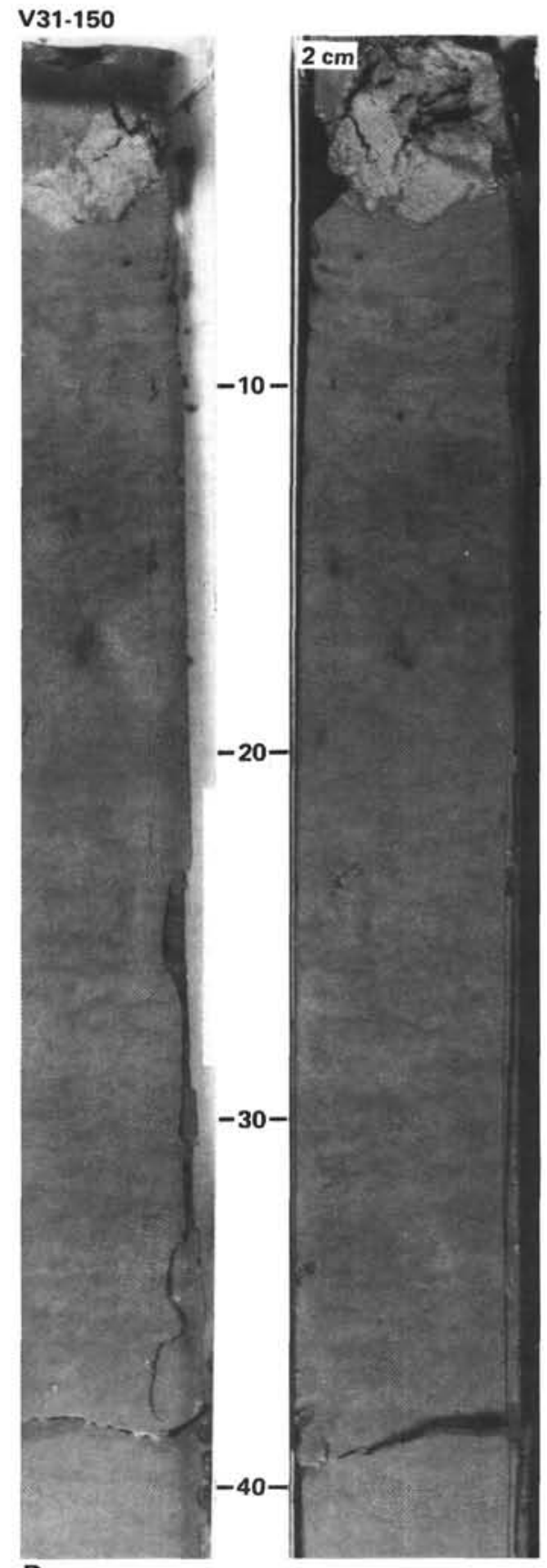

B

Figure 6. Photographs of ash layers occurring in A. Piston Core V26-112, from 344 to $362 \mathrm{~cm}$, and in B. Piston Core V31-150, from 4 to $8 \mathrm{~cm}$, collected within the pelagic clay facies. (The ash layer shown in Piston Core V31-150 is representative of the majority of those recovered from cores in the Lesser Antilles. See the legend in Fig. 1 [back pocket] for cruise identifiers.)

$60 \%$ calcareous microfossils, small percentages of dispersed ash, and only traces of terrigenous silt. Cored ash layers are rare and range in thickness from 1 to $3 \mathrm{~cm}$. No volcanogenic or terrigenous sands or turbidites were recovered. Sediment accumulation rates of 1.67 to $3.33 \mathrm{~g} /\left(\mathrm{cm}^{2} \cdot 10^{3} \mathrm{y}\right.$. $)\left(2-4 \mathrm{~cm} / 10^{3} \mathrm{y}\right.$. $)$ are estimated for this region (see Wright, this volume; Damuth, 1977).

\section{(IV) Volcanic Calcareous Ooze Facies: Broad Arc Platform}

This facies occurs on the shallow northern arc platform (Fig. 4). The bioturbated, light yellowish brown to white volcanic calcareous oozes are characterized by abundant calcareous microfossils and volcanogenic silt, with only minor amounts of clays. The high carbonate content results from the shallow water depths, great distance from South American terrigenous sediment sources, and lack of significant volcanic yield from the Limestone Caribbees (Fig. 5).

Piston cores contain abundant ash layers and volcanogenic calcareous turbidites (Fig. 3). The ash layers are composed of pumice fragments, ash particles, and plagioclase feldspar crystals; thicker layers may display normal size grading. The turbidites range from 3 to $40 \mathrm{~cm}$ 


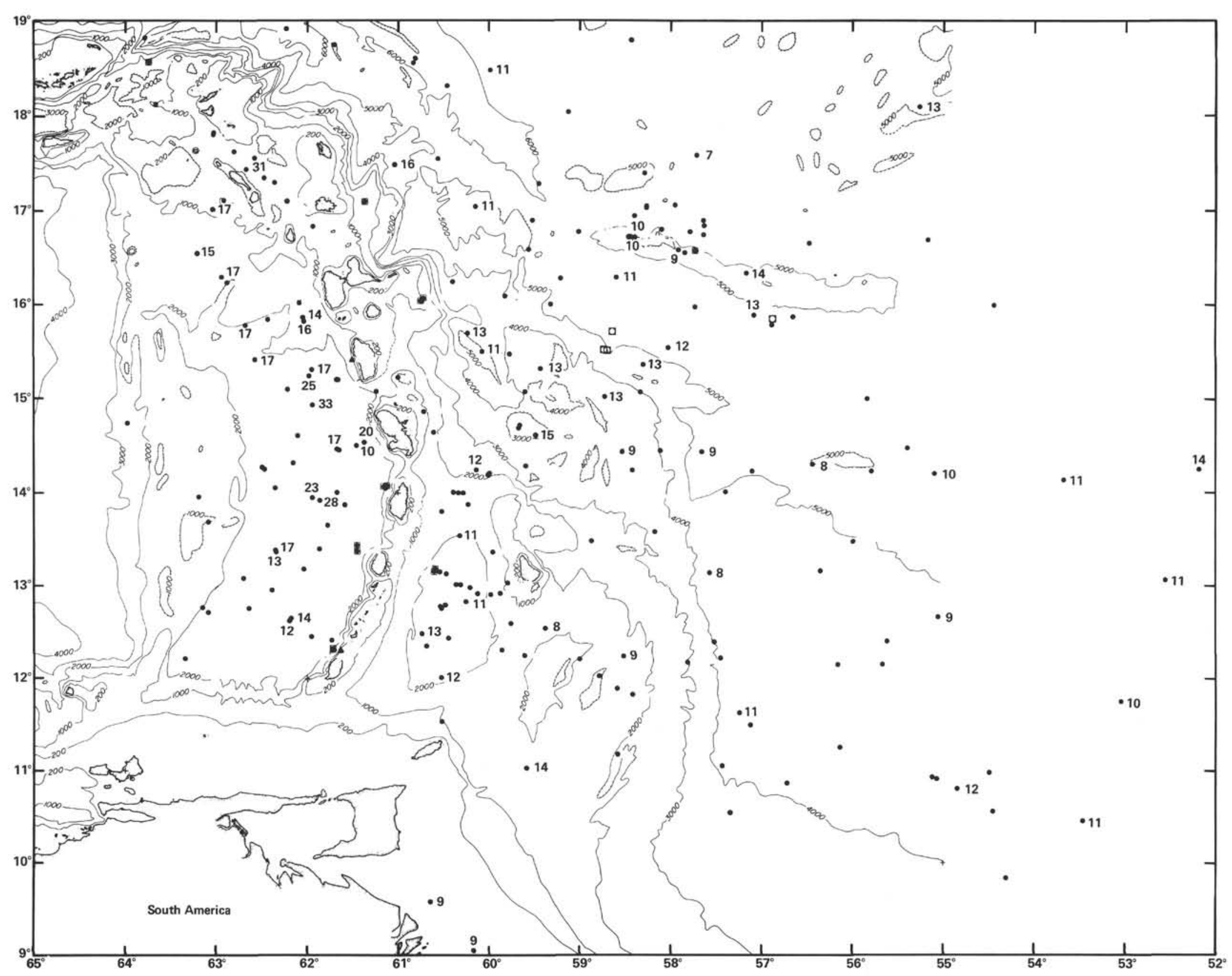

Figure 7. Relative percentage of montmorillonite from surface sediments in selected piston cores from the Lesser Antilles intraoceanic island arc. (The data are listed in Table 3.) 

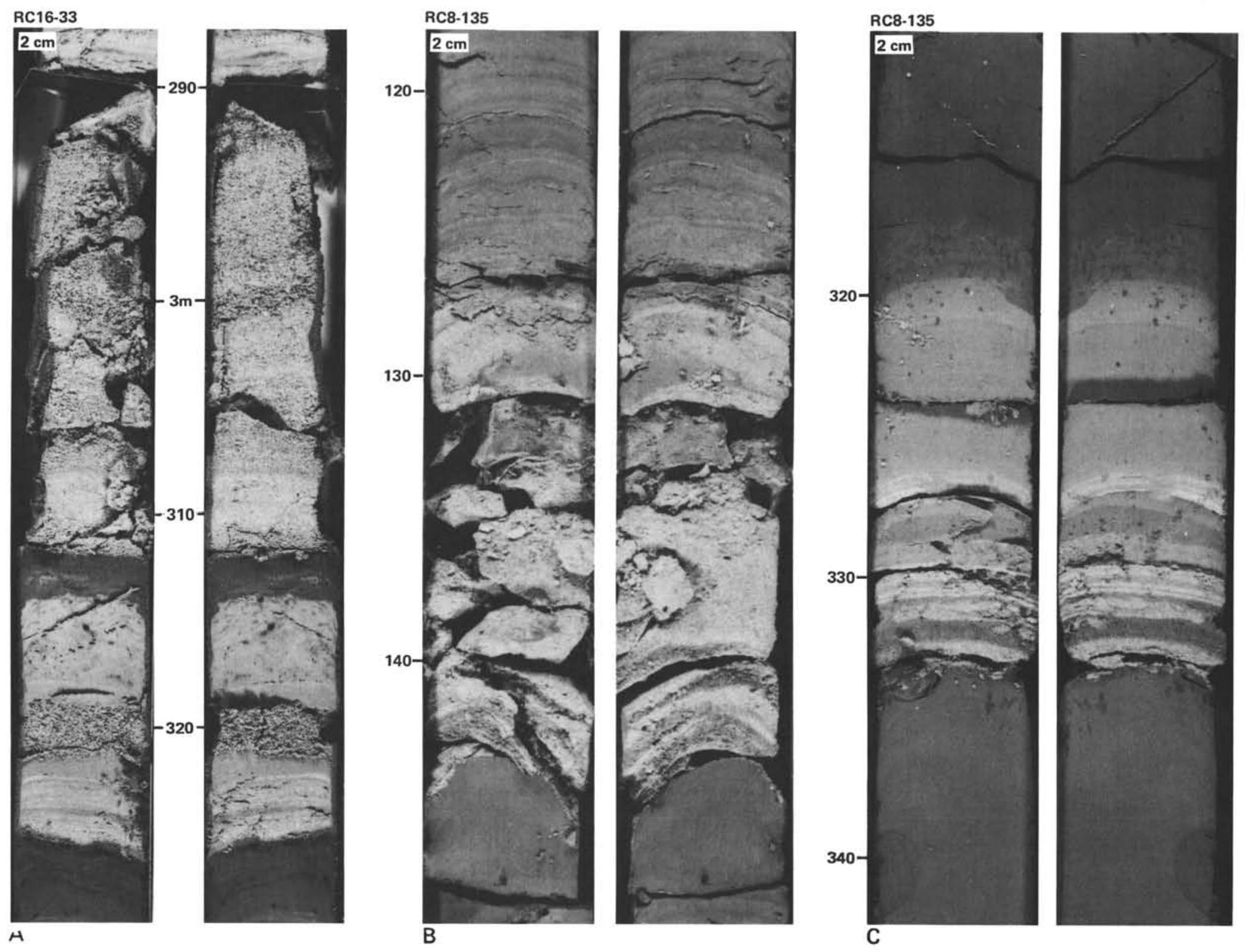

Figure 8. Photographs of graded, laminated calcareous sand layers in A. Piston Core RC16-33, from 287 to $330 \mathrm{~cm}$, in B. Piston Core RC8-135, from 126 to $144 \mathrm{~cm}$, and in C. Piston Cors RC8-135, from 320 to $344 \mathrm{~cm}$, collected within the pelagic clay facies. (These layers were deposited from sediment gravity flows transported down canyons from the adjacent island arc platform. See the legend in Fig. 1 [back pocket] for cruise identifiers.) 
in thickness, show sharp bases and bioturbated tops, and are composed of roughly equal amounts of calcareous shell and volcanogenic rock and crystal fragments (Fig. 9). Quartz is rare or absent. The thicker turbidites are normally graded, and some contain partial Bouma sequences (Bouma, 1962).

\section{(V) Calcareous Shell Hash Facies: Arc Platform Reefs}

This facies unit is characterized by $100 \%$ shell and coral fragments that range in size from silt to very coarse sand or gravel (Figs. 3, 4, and 10). The sand- and gravel-

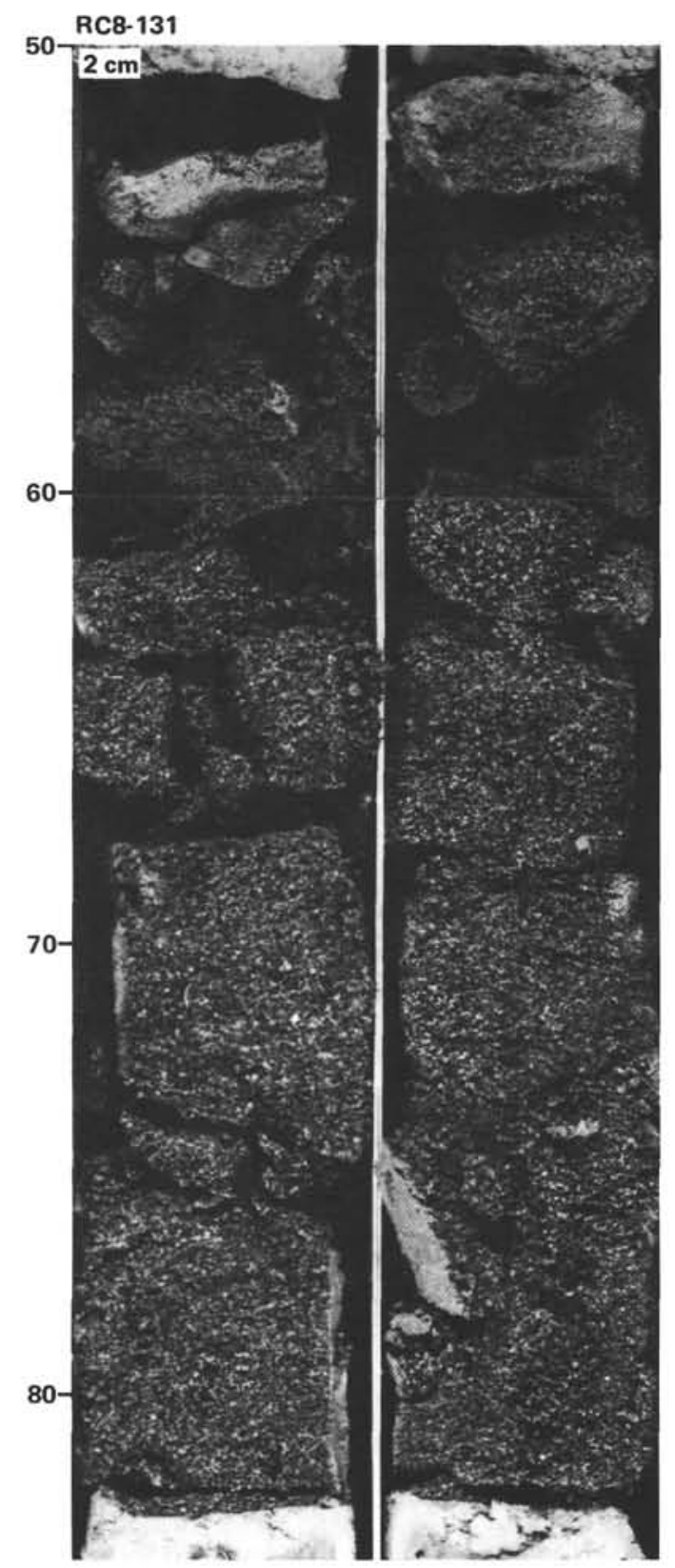

Figure 9. Photograph of a volcanogenic calcareous turbidite in Piston Core RC8-131, from 53 to $83 \mathrm{~cm}$, collected on the west side of the island arc platform within the volcanic calcareous ooze facies. (The coarse-grained calcareous and volcanogenic material was derived by downslope movement from the island arc platform. See back-pocket Fig. 1 for cruise identifier.)

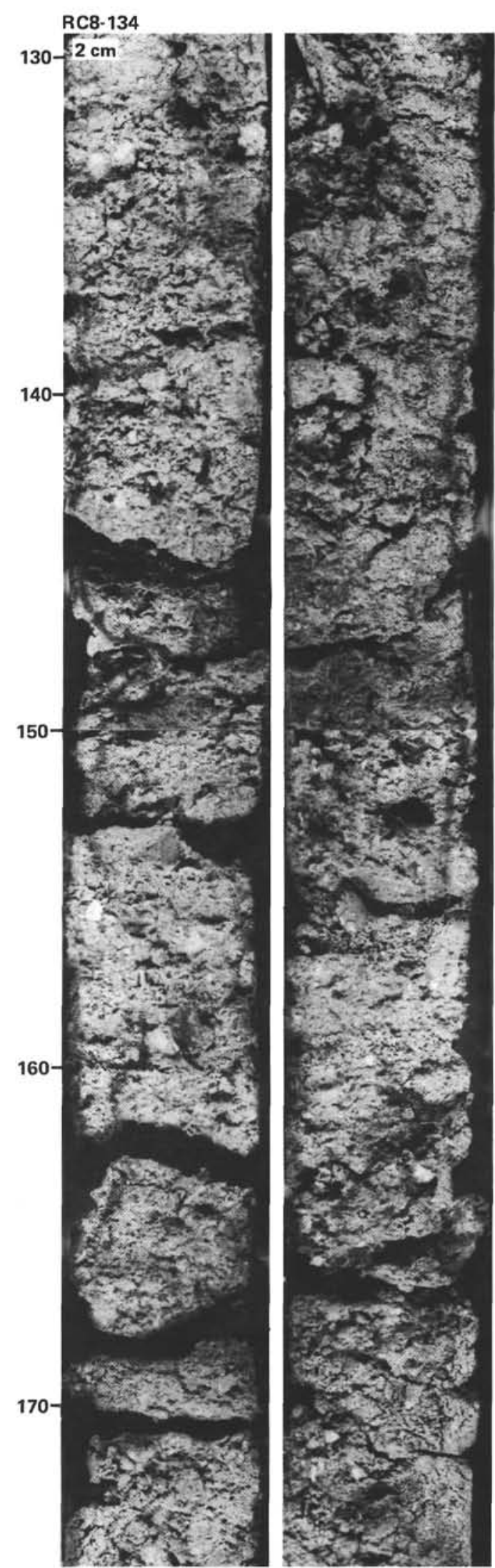

Figure 10. Photograph of material recovered in Piston Core RC8-134, $129-175 \mathrm{~cm}$ from the calcareous shell hash facies. 
sized material consists of coral, bryozoan, pelecypod, and gastropod fragments; the finer fraction consists dominantly of foraminifers with lesser amounts of small gastropods, pelecypod shells, and sponge spicules. This unit is restricted to biologically active shallow-marine environments, such as local bathymetric highs or reefs on the Lesser Antilles arc platform (Keller et al., 1972).

\section{(VI) Calcareous Volcanic Silt Facies: Backarc Basin}

Piston cores from this unit, located in the eastern half of Grenada Basin (Fig. 4), contain olive gray bioturbated silts and sandy silts. These silts are composed of abundant volcanic glass, detrital feldspar, calcareous microfossils, and minor amounts of clay. They are interbedded with numerous pyroclastic turbidites and volcanic sand layers (Fig. 3). Distribution patterns and sedimentary components indicate that the source area for most of the sediment is the Lesser Antilles island chain. The low carbonate percentages (Fig. 5), high montmorillonite content (Fig. 7), and high sediment accumulation rates (see Wright, this volume) result from great influxes of volcanic detritus from the adjacent island arc.

Coring attempts close to the island arc were often unsuccessful because of the coarse-grained nature of the sediments there. The average grain size of sediments in this region has therefore probably been significantly underestimated (Sigurdsson et al., 1980). Pyroclastic turbidites recovered in cores farther from the islands are normally graded, poorly sorted, show sharp bases and bioturbated tops, range in thickness from 4 to $85 \mathrm{~cm}$, and contain up to $25 \%$ clay-size matrix material. The basal portions of these turbidites include clay rip-up clasts, pumice, and charcoal fragments up to $5 \mathrm{~cm}$ in diameter. Cored volcanic sands are well-sorted and ungraded, show sharp bottom and top contacts, range in thickness from 5 to $240 \mathrm{~cm}$, and are composed of subrounded to wellrounded pumice clasts. Ash layers are less abundant than on the east side of the island arc and average only $1 \mathrm{~cm}$ thick.

\section{(VII) Silty Calcareous Mud Facies: Backare and Forearc Regions Adjacent to the Continent}

This unit is found in the southern and western portions of backarc Grenada Basin and in Tobago Trough forearc basin (Fig. 4). Sediment sources include both the island arc and the South American continent. Cores consist mostly of bioturbated, olive gray to grayish brown silty calcareous mud. The silt component of these muds is dominantly of terrigenous origin (mostly quartz with trace amounts of opaques and plant debris).

The muds are interbedded with pyroclastic turbidites and ungraded volcanic sands in the western and southern portions of Grenada Basin and with ash layers in Tobago Trough (Fig. 3). The Grenada Basin pyroclastic turbidites are similar to but less abundant and thinner than those of the calcareous volcanic silt facies. Ash layers in Tobago Trough range from 1 to $29 \mathrm{~cm}$ thick but average only $2 \mathrm{~cm}$ thick. Tobago Trough cores contain no pyroclastic turbidites and only rare thin (less than $5 \mathrm{~cm}$ ) volcanic sands. Cores taken near the island arc and Barbados contain moderately sorted, ungraded calcareous sands derived by downslope movement from adjacent shallow regions (Fig. 3).

The carbonate content of surface sediments increases toward the northern end of Grenada Basin because of decreasing influx of South American sediment. Tobago Trough cores are characterized by very low carbonate contents, reflecting extensive dilution by influx of both island arc volcanogenic and South American terrigenous sediment (Fig. 5). High carbonate contents reported immediately east of Grenada Island are the result of slumping and downslope transport of skeletal debris from reefs adjacent to the Grenadine Islands (Keller et al., 1972).

Montmorillonite percentages are greater in Grenada Basin than in Tobago Trough, reflecting larger volcanic influxes to the west from the island arc (Fig. 7). Illite percentages are greater in the southern part of the lithofacies area, especially in Tobago Trough, closer to Orinoco River sediment sources (Fig. 11).

Seismic profiles reveal that the smooth seafloor in the southern part of Tobago Trough is broken by submarine valleys that extend into the basin from the South American continental margin. Some portion of the terrigenous detritus found in the forearc basin was undoubtedly transported there via these valleys (Keller et al., 1972), although no turbidites have been recovered in piston cores from that region.

\section{(VIII) Calcareous Mud Facies: Abyssal Plain Adjacent to the Continent}

Cores collected from within this facies unit, located above the CCD on the abyssal plain near South America (Fig. 4), consist of a Holocene light brown bioturbated pelagic-hemipelagic calcareous mud with small amounts of dispersed terrigenous (mostly quartz) and volcanogenic silt. These calcareous muds overly a Pleistocene olive gray to gray hemipelagic unit that contains interbeds of dark grayish brown terrigenous silt and sand (Fig. 3). The contact between these units is marked typically by a hard, rust-colored, iron-rich crust. This crust developed at the sediment/water interface by oxidation and precipitation of dissolved reduced iron after cessation of Pleistocene terrigenous turbidite deposition (McGeary and Damuth, 1973). Illite contents are high due to close proximity to illite-rich Orinoco River sources (Fig. 11; Nota, 1958; Embley and Langseth, 1977; Eisma et al., 1978). Montmorillonite percentages are correspondingly low (Fig. 7).

The terrigenous silt and sand layers, derived from South American sources, were deposited as both turbidites and contourites (Damuth, 1977). The turbidites are normally graded and poorly sorted with a 10 to $30 \%$ clay matrix, show sharp bottom and bioturbated top contacts, and range from 3 to $57 \mathrm{~cm}$ in thickness (Fig. 12A). They are composed of abundant angular to subangular quartz grains with smaller amounts of plagioclase and organic matter; garnet, volcanic glass, manganese micronodules, and reworked biogenic material occur in trace amounts. The darker-colored layers contain greater per- 


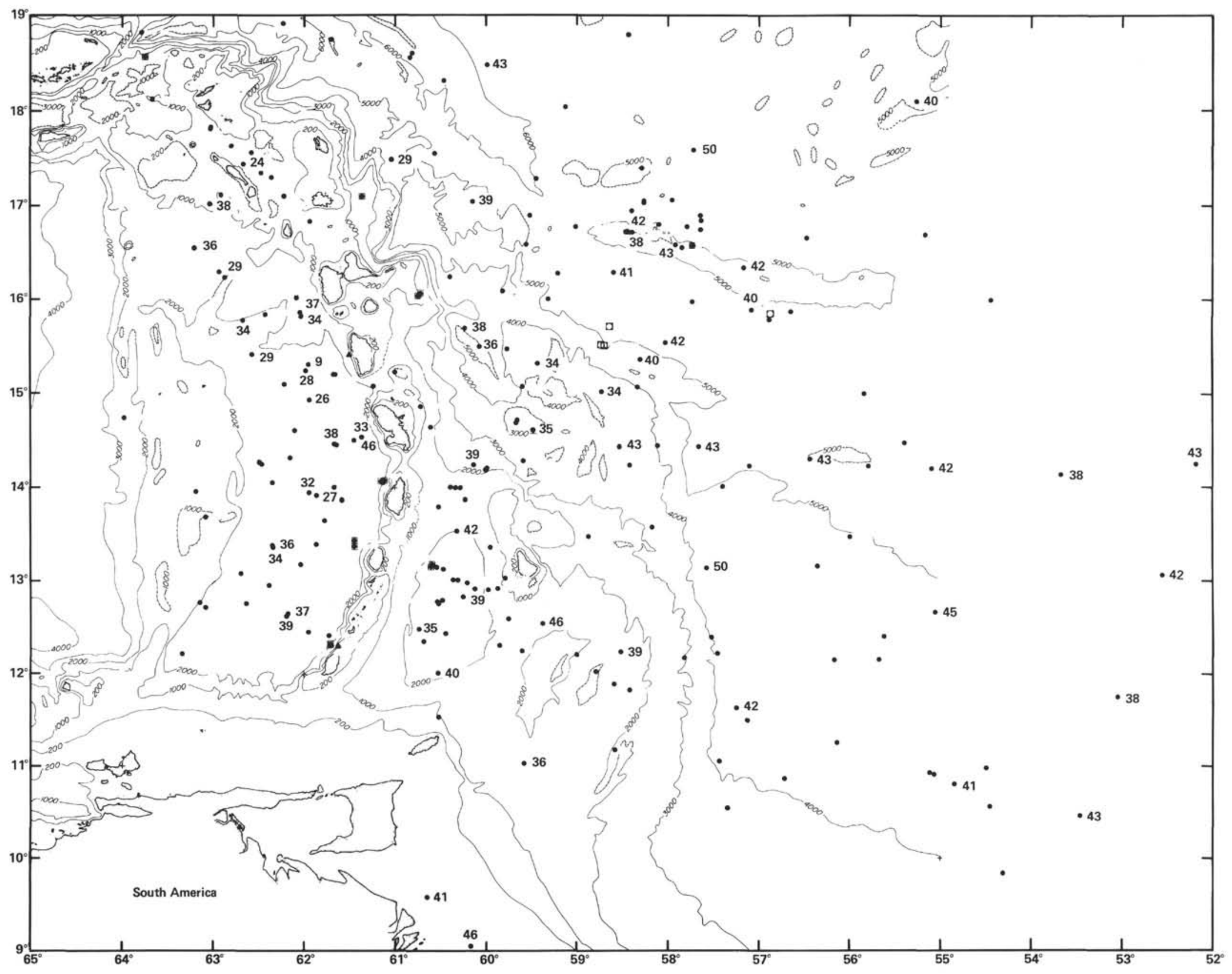

Figure 11. Relative percentage of illite from surface sediments in selected piston cores from the Lesser Antilles intraoceanic island arc. (The data are listed in Table 3.) 

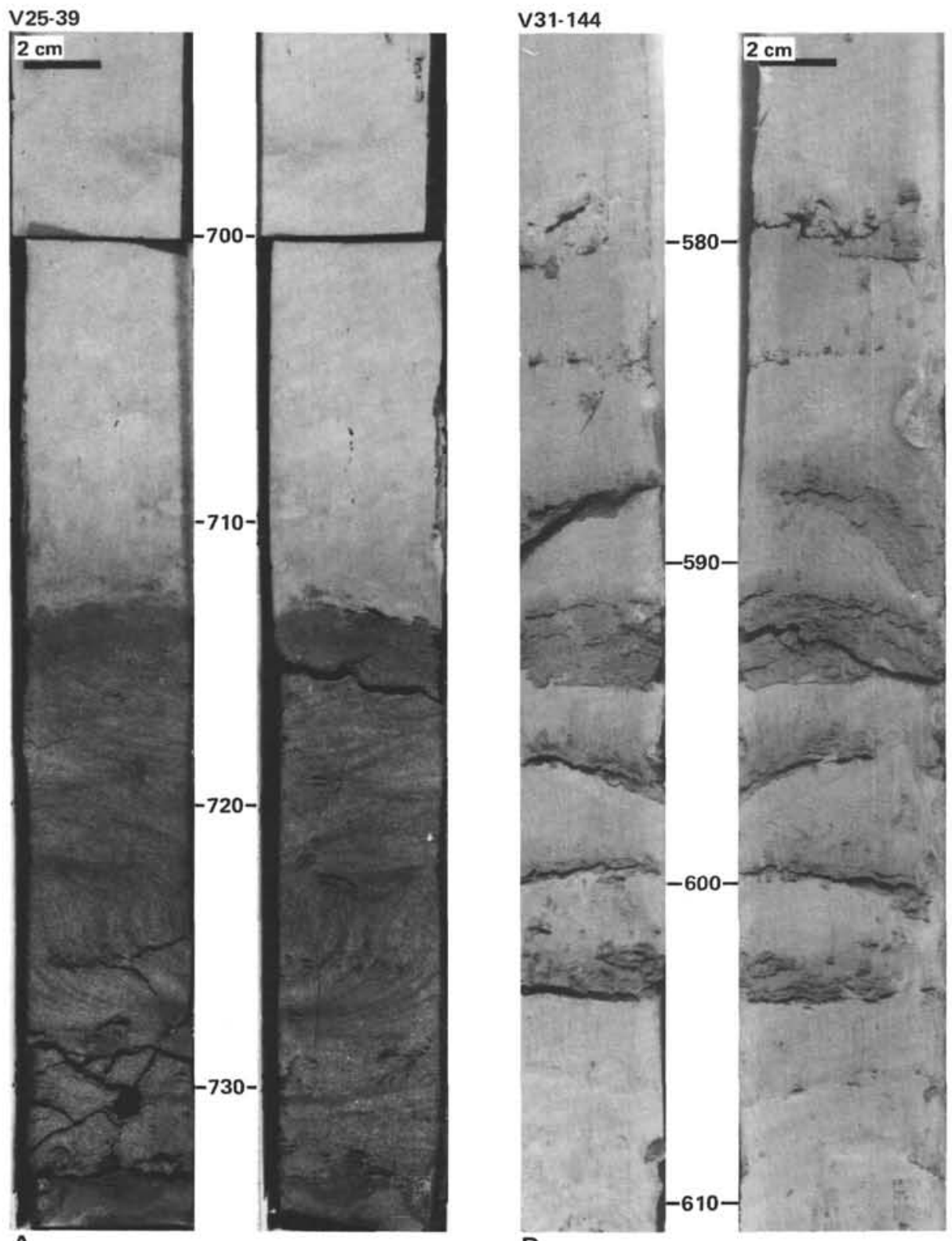

A

B

Figure 12. A. Graded, cross-bedded terrigenous turbidite layer in Piston Core V25-39, from 712 to $740 \mathrm{~cm}$, collected from the calcareous mud facies on the Atlantic abyssal plain close to the South American continent. (The terrigenous silt and sand were derived from continental sediment sources.) B. Contourites in Piston Core V31-144, from 570 to $612 \mathrm{~cm}$, collected within the calcareous mud facies immediately north of the Demerara Plateau. (These contourites are thin, ungraded, well-sorted, and show sharp bottom and top contacts.)

centages of organic debris, mostly small pieces of leaves and wood. The thickness, abundance, and grain size of these turbidite layers decreases away from the South American continent (Fig 3).

The contourite layers are ungraded, well-sorted, contain very little clay-size matrix, range from 0.5 to $2 \mathrm{~cm}$ thick, show sharp bottom and top contacts, and are composed of subrounded, quartz-rich coarse silt to very finegrained sand (Fig. 12B). Contourite deposits are limited to the region immediately north of the Demerara Plateau (Fig. 3; Damuth, 1977).

\section{DISCUSSION OF FACIES DISTRIBUTION}

\section{Nondiagnostic Sedimentary Components}

The facies units delineated in this study are distinguished from one another by characteristic textural and compositional differences. It is important, however, to point out that some sedimentary components occur in all the cores recovered and are therefore not diagnostic in any facies classification scheme. Siliceous microfossils and dispersed volcanic ash both fall into this category. Siliceous microfossils are found in all the Lesser 
Antilles piston cores, though they are most prevalent in the pelagic clay facies cores where their abundance is not diluted by calcareous microfossils. Dispersed volcanic ash is also a component in all the cores, though it is most abundant in cores from the forearc side of the arc platform.

\section{Clay Mineralogy}

Of the four major clay mineral groups identified, only montmorillonite and illite show characteristic areal trends. Montmorillonite is derived principally from the breakdown of volcanic material, and the percentage of montmorillonite decreases with increasing distance from the island arc (Fig. 13). Percentages of illite are greater in the Lesser Antilles forearc than in the backarc region (Fig. 14). Most of this illite, derived from the Orinoco River, is transported north to forearc depositional sites by ocean currents (see Ocean Current section that follows later in the chapter). This trend is likely more pronounced at low stands of sea level when greater amounts of terrigenous material bypass shelfal areas.

\section{Acoustic Facies Mapping Correlation with Lithofacies Units}

Damuth (1975) divided the western equatorial Atlantic seafloor, including the Lesser Antilles forearc region, into acoustic facies provinces based on variations in highfrequency $(3.5-12 \mathrm{kHz})$ seismic response. He related acoustic echo character to coarseness of sediment and concluded that there is a distinct decrease in the abun- dance and grain size of silt and sand layers from south to north on the Atlantic abyssal plain adjacent to the Lesser Antilles intraoceanic arc. The core data from the calcareous mud and the pelagic clay facies described earlier support his conclusions.

\section{CONTROLS ON FACIES DISTRIBUTION}

\section{Local Level of the CCD}

The location of the modern CCD, as distinguished by the downslope disappearance of calcareous microfossils in pelagic or hemipelagic surface sediments, defines the boundary between the calcareous mud or marly calcareous ooze facies and the noncalcareous pelagic clay facies. This boundary is considered to be at $10 \%$ carbonate content (see Introduction and Explanatory Notes for Leg 78A, this volume). Calcareous components are present in surface sediments below the CCD as reworked components incorporated in turbidites and debris flows. The surface sediment carbonate data from the Lesser Antilles region indicates that the modern CCD is at about $5200 \mathrm{~m}$ (Fig. 15). The boundary between the calcareous mud or marly calcareous ooze facies and the noncalcareous pelagic clay facies will shift upslope or downslope as the CCD fluctuates with time (van Andel, 1975).

\section{Local Wind Patterns}

Distribution of volcanic ash layers in the vicinity of the Lesser Antilles arc is controlled by local wind pat-

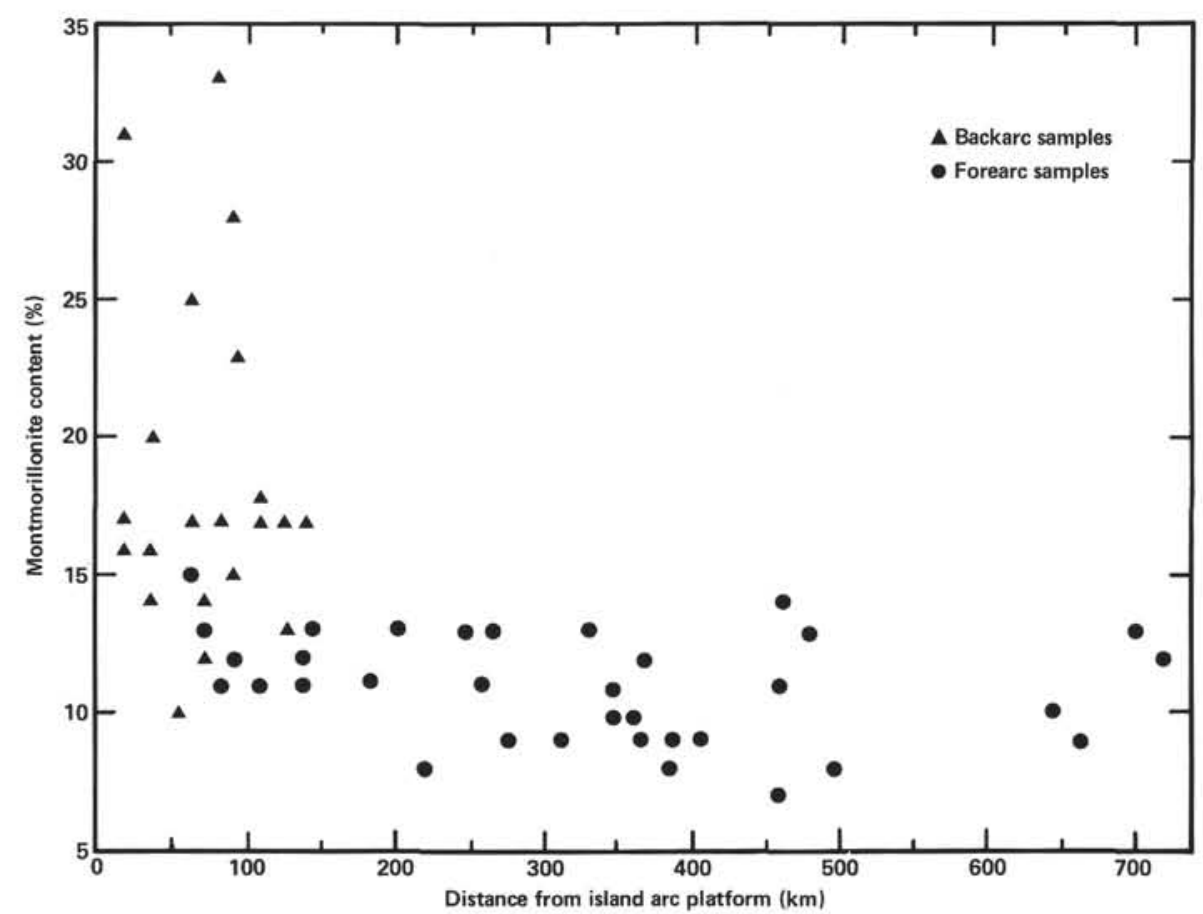

Figure 13. Plot of montmorillonite percentage in piston-core surface sediments versus distance from island arc platform volcanic sources. (Triangles represent backarc samples from Grenada Basin and the Aves Ridge; circles represent forearc samples from the accretionary prism and Atlantic abyssal plain. The montmorillonite percentage present in these surface samples decreases with increasing distance from the island arc platform both east and west of the arc.) 


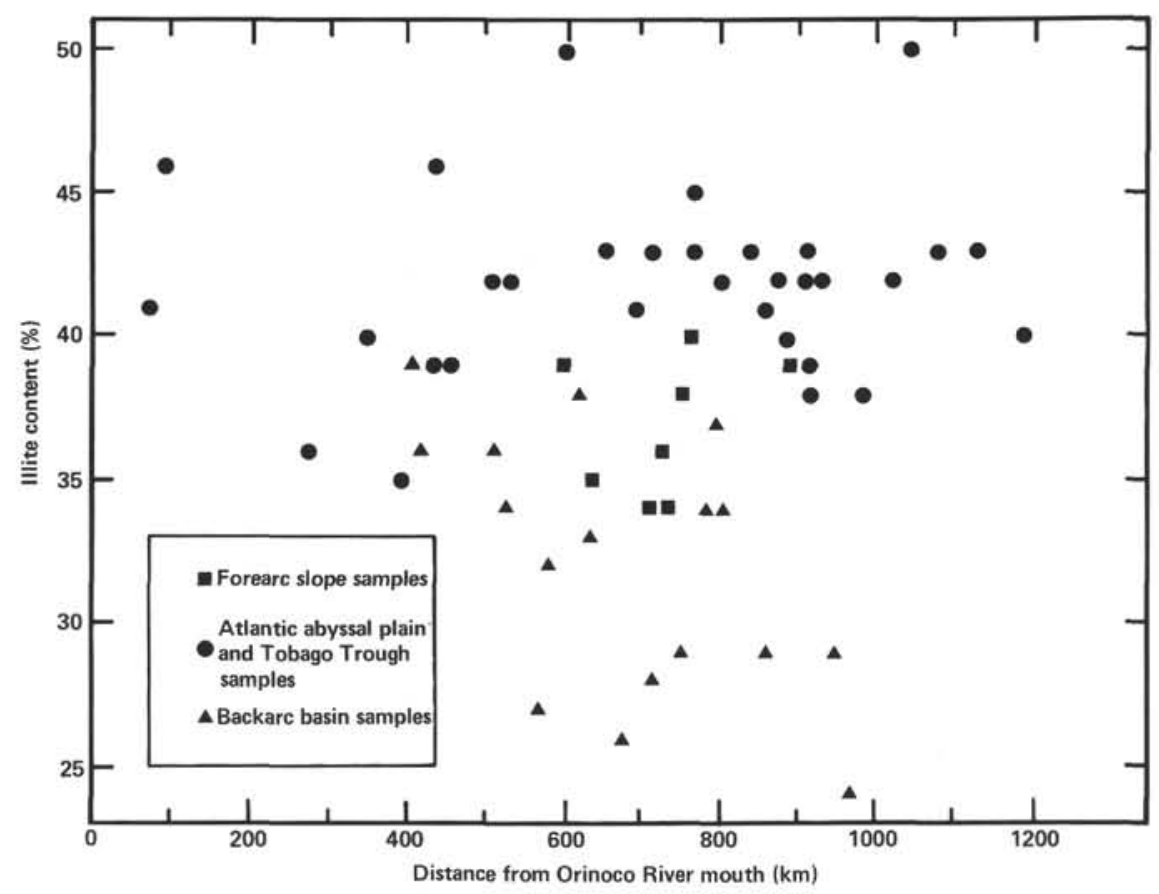

Figure 14. Plot of illite percentage in piston-core surface sediments versus distance from the South American Orinoco River mouth. (Backarc basin samples contain less illite than forearc samples.)

terns. This region is dominated by a layered atmospheric structure that varies with the seasons (Newell et al., 1972). The surface winds are the easterly trades that travel at velocities of about $20 \mathrm{~km} / \mathrm{hr}$. These winds extend to 5 to $8 \mathrm{~km}$ above sea level, depending on the time of year. The westerly winds lie above the easterly trades, extend to about $20 \mathrm{~km}$ above sea level, and travel at velocities ranging from 50 to $90 \mathrm{~km} / \mathrm{hr}$. Winds in the overlying stratosphere travel to the west at velocities of about $20 \mathrm{~km} / \mathrm{hr}$.

Distribution of a given eruption cloud of ash depends on the height of the cloud, thereby determining which wind system transports that body of ash. Evidence from recent eruptions indicates that the vast majority of the Lesser Antilles ash plumes are carried east into the Atlantic Ocean basin by the westerlies (Sigurdsson et al., 1980 ). Evidence from cores collected in the Lesser Antilles indicate that these wind patterns have been operating throughout the Quaternary, as cores taken in the forearc region contain a greater percentage of airfall ash layers than do cores collected from the backarc region (Fig. 3).

\section{Ocean Current Patterns}

Surface and bottom ocean currents in the Lesser Antilles region are effective in transporting both volcanogenic detritus from the island arc and terrigenous detritus from the South American continent. The Western Boundary Undercurrent flows south or southeast along the Lesser Antilles slope at velocities of about 1 to $3 \mathrm{~cm} / \mathrm{s}$ (Fig. 2; McCoy, 1969); this current shapes the contourites observed in cores collected immediately north of the Demerara Plateau. There are also two surface current systems operating in the region, both of which exert strong influence on the distribution of sediment in the area. The Equatorial Current flows west through island passages along the arc chain at velocities of 20 to $30 \mathrm{~cm} / \mathrm{s}$, and the Guiana Current flows northwest along the South American continental shelf (Fig. 2; Metcalf, 1976). The Equatorial Current transports ash fallout back toward the island arc and also carries volcanic sands deposited in the shallow island passages westward into Grenada Basin (Sigurdsson et al., 1980). The Guiana Current carries clay and fine-grained silty terrigenous detritus from South American river sources northwest along the South American continental shelf into the Lesser Antilles forearc region. The dispersed terrigenous detritus found in the silty calcareous mud facies was transported in this fashion.

\section{Distance from South American Sediment Sources}

Terrigenous sediment from South America is deposited in the Lesser Antilles region in two ways. First, finegrained silt and clay are carried into the region by the Guiana Current system and contribute to the hemipelagic component of the sediments. Second, coarser-grained silt and sand periodically are funneled as turbidites down the South American continental slope and deposited on the Atlantic abyssal plain. These turbidites are reworked and redeposited as contourites in the region north of the Demerara Plateau.

Figure 16 shows schematic lithologic sections for Atlantic abyssal plain and accretionary prism sediments in both the southern and northern Lesser Antilles region. These sections indicate that there is significant lateral variability in the sediments deposited within the forearc region. The northern abyssal plain, far from South 


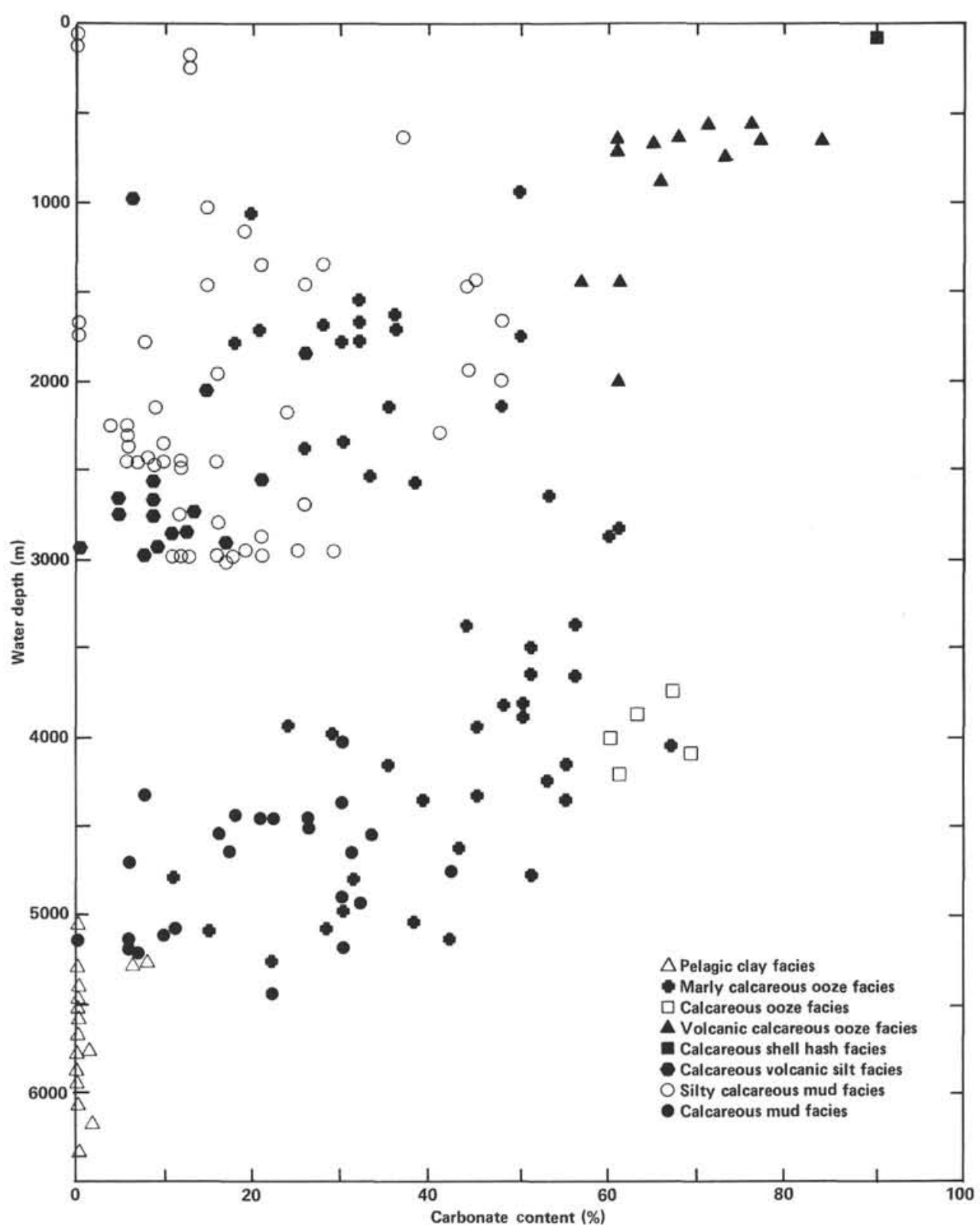

Figure 15. Plot of surface sediment carbonate content versus water depth from piston cores collected in the Lesser Antilles region. (Carbonate contents were determined with the "Karbonate Bombe" [Müller and Gastner, 1971]. The modern CCD is at about $5200 \mathrm{~m}$ water depth.)

American terrigenous sediment sources, is covered by a relatively thin sequence of hemipelagic and pelagic sediments (Fig. 16, Lithologic Section 2). The southern abyssal plain is covered with a 4-km-thick blanket of interbedded hemipelagics and terrigenous turbidites that mask the bathymetric expression of the trench there (Fig. 16, Lithologic Section 4). The dispersed South American terrigenous component and the number and thickness of terrigenous turbidites all increase from north to south. Accretionary prism sedimentary sections consist of offscraped abyssal plain sediments overlain by slope marly calcareous oozes in the north and by silty calcareous muds in the south (Fig. 16, Lithologic Sections 1 and 3 ).

\section{Distance from Island Arc Sediment Sources}

Distance from the island arc influences the amount of volcanogenic material present in Lesser Antilles sediments. Two basic types of volcanogenic sediments are derived from the island arc: (1) volcanic ash, deposited as airfall ash deposits, and (2) coarser pyroclastic sediments, deposited as turbidites or debris flows. Due to the prevailing wind patterns, most of the volcanic ash is deposited on the Atlantic Ocean side of the Lesser Antilles island arc, with the abundance and thickness of the ash layers decreasing to the east. In contrast, most of the coarser pyroclastic material is deposited west of the island arc (Fig. 3). This contrast is due to both the 


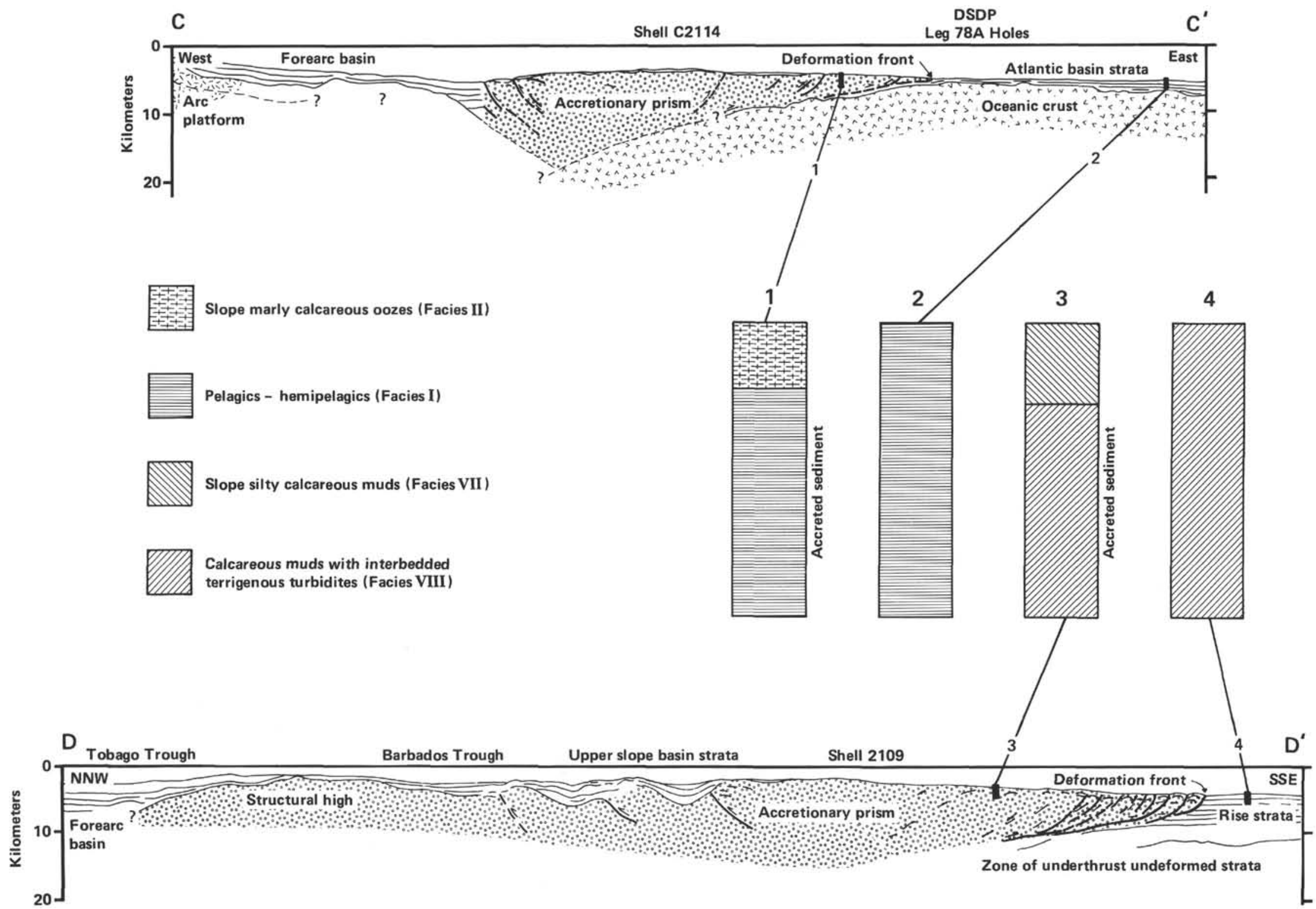

Figure 16. Schematic tectonic cross sections (C-C'; D-D') and lithologic sections (1-4) for the Lesser Antilles intraoceanic island arc. (Tectonic cross sections are modified from R.C. Speed et al., "Lesser Antilles arc system and adjacent terranes," unpublished final report to JOI, Inc., June 30, 1982. Locations of cross sections are shown in Fig. 1. The cross sections have a 2:1 vertical exaggeration. The shallow structure is controlled by multichannel seismic sections, whereas the deeper structure is controlled by refraction data. Note increase in size of forearc basin, width and thickness of accretionary prism, and thickness of incoming sedimentary section on the Atlantic abyssal plain from the northern [C-C'] to the southern [D-D'] section.) 
ocean surface current patterns and the steeper slopes of the backarc side of the arc platform (Sigurdsson et al., 1980). The abundance and thickness of pyroclastic turbidites decreases to the west (Fig. 3). Moreover, montmorillonite percentages, which also reflect an arc source, decrease away from the arc in both the forearc and the backarc regions (Fig. 13).

\section{Bathymetry}

Local bathymetry greatly influences sediment distribution in the Lesser Antilles region. The Atlantic abyssal plain is dissected by the Barracuda Rise and the Tiburon Rise (Fig. 2). These oceanic ridges influence regional sediment distribution patterns in two ways. First, the tops of the ridges are covered exclusively with hemipelagic and pelagic sediments because they are isolated both from terrigenous material coming north from South America and from biogenic or volcanogenic turbidites or debris flows from the accretionary prism slope. The lack of terrigenous turbidites at Site 543 is likely due to its location on the Tiburon Rise. Second, the ridges serve as barriers to South American terrigenous sediment coming north along the deformation front. The decrease in sediment thickness on the Atlantic abyssal plain north of the Tiburon and Barracuda rises is likely due to the damming effects of those rises (Chase and Bunce, 1969; Peter and Westbrook, 1976; Fig. 17).

Another bathymetric factor is the slope angle. The average slope west of the Lesser Antilles island arc platform into Grenada Basin is $9^{\circ}$, whereas the slope east of the arc averages only $1.5^{\circ}$ (Sigurdsson et al., 1980). This difference provides a possible explanation for the concentration of pyroclastic deposits on the western side of the island arc. Steep slopes are necessary to generate sediment-gravity flows and the eastern side of the arc is characterized by insufficient relief to initiate significant downslope movement. Debris flows that enter the ocean on the east side of the island chain tend to slow and deposit their loads before they get far from the arc (Si-

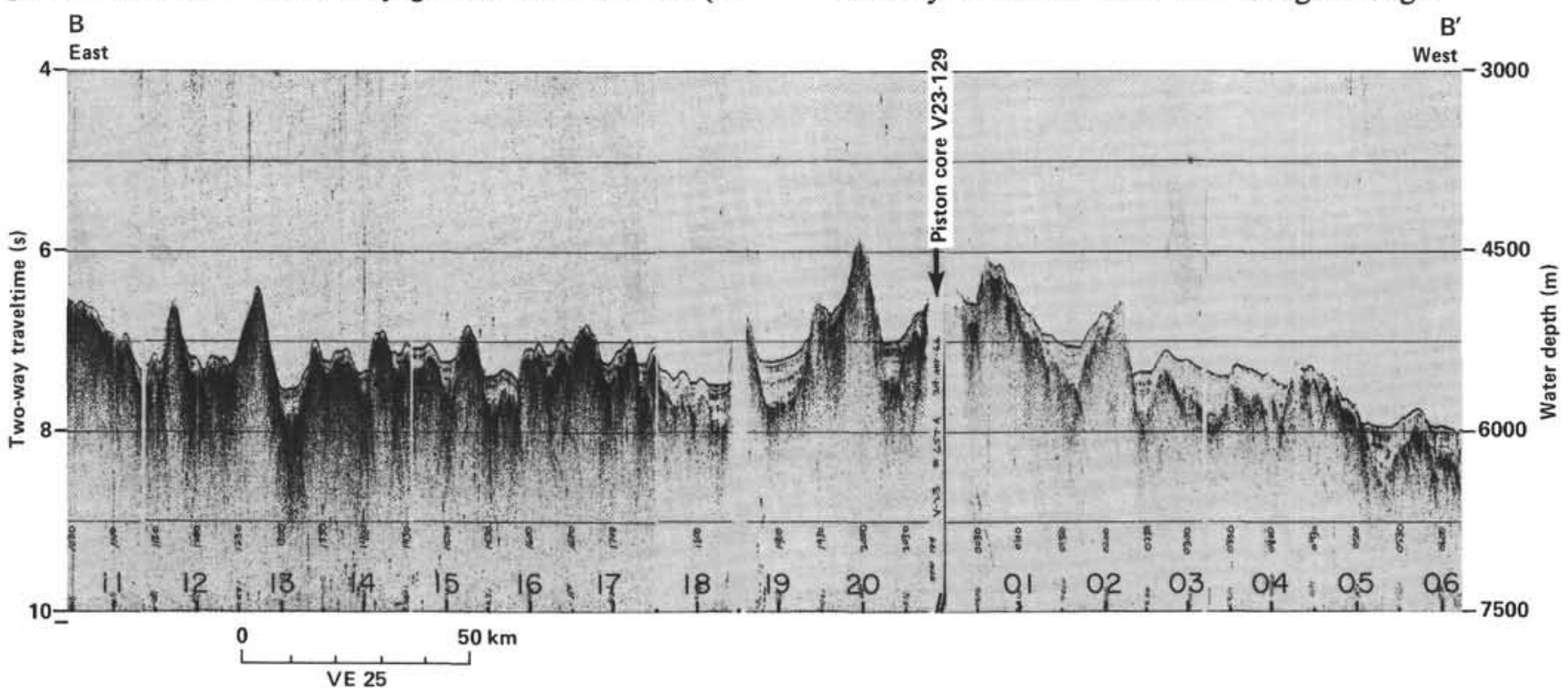

Figure 17. Airgun profile B-B' across the Atlantic abyssal plain north of the Barracuda Rise. (Location of profile is shown in Fig. 1. Sediment cover is thin due to damming effects of Barracuda Rise and distance from South American sediment sources.)

gurdsson et al., 1980). Coarse-grained sediments that travel down the slope of the accretionary prism do so via submarine canyons, as indicated by the calcareous sands recovered in canyons in the northern part of the study area.

\section{Variations in Sea Level}

Because of the Holocene relative high stand of sea level, coarse-grained terrigenous sediment from the Orinoco River is trapped on the South American continental shelf (Nota, 1958). The only present-day sediment contribution from South America is clay and very fine silt, which is transported northwest along the South American shelf by the Guiana Current system. The terrigenous turbidites of the calcareous mud facies were deposited during low stands associated with Quaternary glacial periods, when South American rivers debouched on the outer continental shelf (Damuth, 1977).

\section{DEPOSITIONAL MODEL}

The following model, based upon the distribution of sediment facies units, summarizes the depositional processes operating in the Lesser Antilles intraoceanic island arc (Fig. 18). Thick sediment accumulations are restricted to backarc Grenada Basin, Tobago Trough forearc basin, and the abyssal plain adjacent to the South American continental slope.

During low stands of sea level, coarse-grained terrigenous sediments from South America are transported as sediment-gravity flows down submarine canyons to abyssal plain depositional sites. These flows build a thick sediment wedge of hemipelagic clays and interbedded turbidites on the Atlantic abyssal plain close to the South American continent (Fig. 18). The thickness of the wedge, the terrigenous component of the sediments, and the thickness and width of the resulting accretionary prism all decrease to the north (Fig. 16). Canyons similarly funnel material into Tobago Trough. 


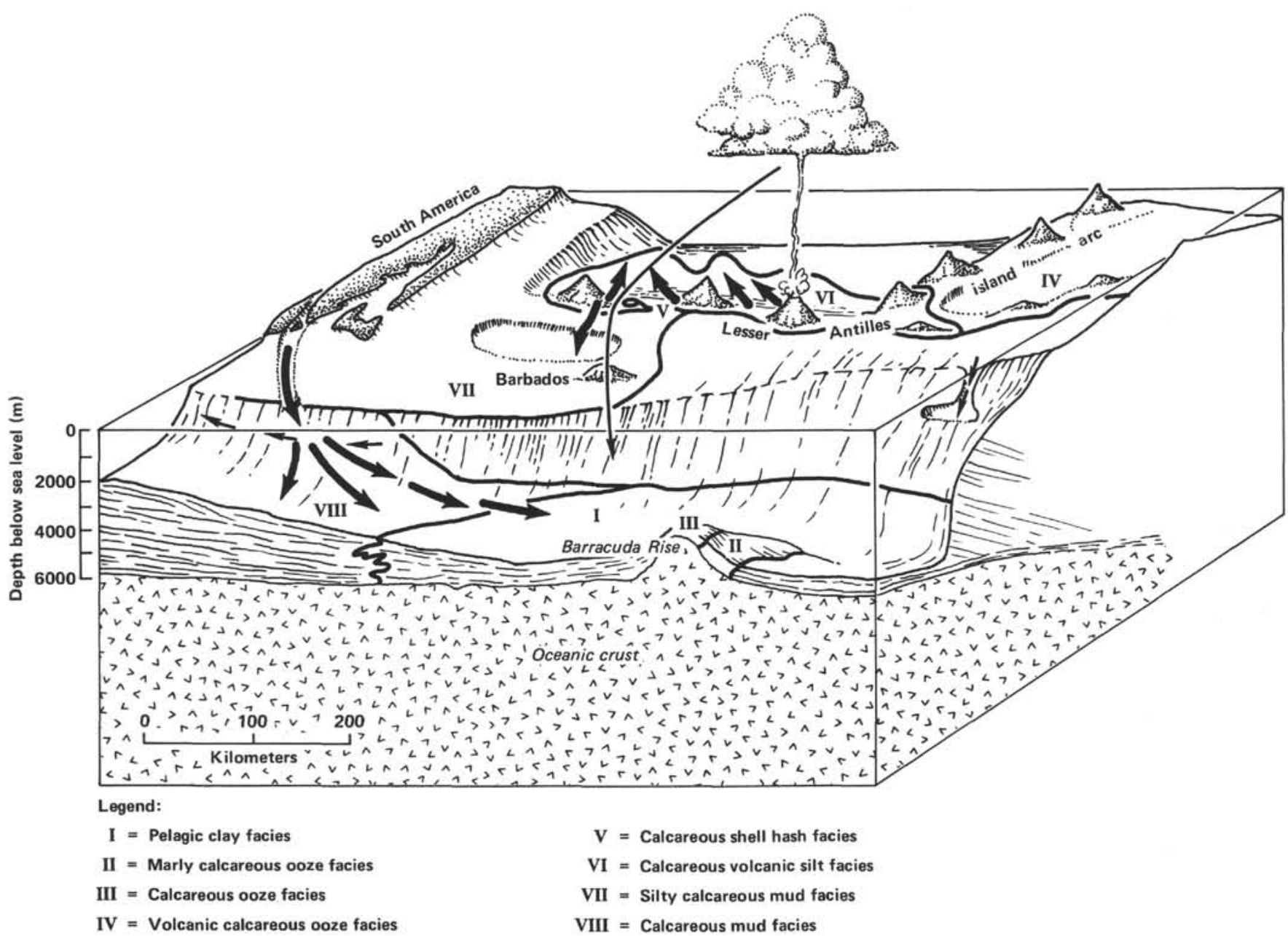

Figure 18. Depositional model for the Lesser Antilles, an intraoceanic island arc with a lateral source of terrigenous sediment, showing lithofacies unit distribution. (Thick arrows from the island arc platform into the backarc basin indicate dominant direction of transport of volcanogenic debris; thick arrows from the South American Orinoco River mouth indicate direction of transport of terrigenous silt and sand via turbidity currents. Thin arrows along the continental slope parallel to bathymetric contours indicate reworking and redeposition of continentally derived silt and sand by contour currents.)

Submarine canyons are cut off from sediment supplies during sea-level high stands. As a result, modern interglacial terrigenous sand and silt are restricted to depositional sites on the South American shelf. Only the finest terrigenous clay and silt are carried beyond the shelf edge by ocean surface currents, and the adjacent slope and abyssal plain are blanketed with a Holocene layer of calcareous mud. The relatively larger volume of terrigenous sediment that is contributed to trench floor and abyssal depositional sites at low stands of sea level is characteristic of active margin settings in general (Scholl and Marlow, 1974; Scholl et al., 1977; Underwood et al., 1980).

Coarse-grained volcanogenic detritus derived from the island arc is transported west and deposited in the backarc basin as pyroclastic turbidites (Fig. 18). This deposition is the result of the steeper flanks of the backarc side of the arc, the location of the active volcanic centers on the western side of the arc platform, and the western flow of the Equatorial Current through island arc passages. With the exception of the Equatorial Current pattern, which is specific to the Lesser Antilles region, these factors are applicable to intraoceanic island arcs in general. Thus as a general rule, coarse-grained volcanogenic material is preferentially deposited in the backarc region of active margin settings.

In contrast, most of the fine-grained volcanic ash in the Lesser Antilles is transported east by the regional winds and deposited on the forearc side of the arc. The direction of ash transport in arc settings is dependent entirely on the regional wind patterns; the distribution patterns in other arc settings may therefore be significantly different.

Calcareous biogenic material is present both as a pelagic component above the CCD and as local turbidites and debris flows in submarine canyons on either side of the arc platform. The abundance of this material is largely controlled by local fluctuations in the level of the CCD and by dilution effects from influxes of volcanogenic and terrigenous detritus. The pelagic biogenic component in island arcs in more northernly latitudes, such as the Aleutian arc, is dominantly siliceous, and the pelagic sediments are correspondingly siliceous muds and oozes. 


\section{CONCLUSIONS}

The depositional model presented in this chapter is likely applicable to all intraoceanic island arcs characterized by significant lateral sources of terrigenous sediment. This model differs from models developed both for continental convergent margins (Piper et al., 1973; Moore, Watkins, et al., 1982; Underwood and Bachman, 1982) and for intraoceanic convergent margins isolated from continental sediment sources (Lundberg, 1983). Data from continental convergent margins indicate that substantial amounts of terrigenous gravel, sand, and silt are eroded from the adjacent continental block and are deposited on the shelf, in intraslope basins, and in trench segments fed by major submarine canyons. Thus continental arc-trench systems contain a much greater volume of coarse detritus than the Lesser Antilles, although many of the depositional processes (e.g., sediment bypassing in submarine canyons) are identical for the two types. Intraoceanic convergent margins without sources of continental terrigenous sediment typically lack thick trench deposits and large accretionary prisms because of substantially lower rates of sedimentation (Hussong, Uyeda, et al., 1982; Lundberg, 1983). It is the "starvation" of the trench floor that distinguishes such systems from the Lesser Antilles type.

It should be emphasized that there is a great deal of variability in the sediment distribution and depositional processes operating in active margin settings. The depositional model presented here for the Lesser Antilles does not entirely account for all the variability observed at intraoceanic island arcs with significant lateral sources of terrigenous sediment. It should, however, prove useful for future researchers investigating both similar modern and ancient uplifted equivalents.

\section{ACKNOWLEDGMENTS}

This chapter was reviewed by George deVries Klein and Michael B. Underwood. Valuable comments throughout the course of manuscript preparation were contributed by Casey Moore, Eli Silver, and Bob Garrison. The crew of the Glomar Challenger were extremely helpful in the collection and shipboard processing of the Leg 78A cores. Sarah Roeske provided much assistance in the preparation of smear slides and clay X-ray diffraction samples. Jim Hein offered frequent and valuable advice on clay X-ray techniques. DSDP provided the "Karbonate Bombe" to run carbonate content measurements on the piston cores. The X-ray diffractometer at the University of California at Santa Cruz was used to run the X-ray samples. Assistance in sampling and describing piston cores was generously provided by Floyd McCoy at LDGO, James Broda at WHOI, and Asaf Ashraf, Steve Carey, and Haraldur Sigurdsson at the University of Rhode Island.

Piston cores at the University of Rhode Island were collected under NSF Grants OCE 75-21197 (Cruise GS-7605) and OCE 77-25789 (Cruise EN-20). The Woods Hole core repository is supported by NSF Grant OCE 82-25231; the Lamont-Doherty repository is supported by NSF Grants OCE 78-25448 and OCE 80-22109. Partial support for this study came from Joint Oceanographic Institutions, Inc. as part of an Ocean Margin Drilling Program geological and geophysical synthesis study of the Lesser Antilles intraoceanic island arc. Additional support was received in the form of a Patent Fund grant from the University of California at Santa Cruz and a Sigma Xi Grant-in-Aid-of-Research from the Sigma Xi Scientific Research Society.

\section{REFERENCES}

Bader, R. G., Gerard, R. D., et al., 1970a. Init. Repts. DSDP, 4: Washington (U.S. Govt Printing Office). 1970b. Site 27. In Bader, R. G., Gerard, R. D., et al., Init. Repts. DSDP, 4: Washington (U.S. Govt. Printing Office), 93-123.

, 1970c. Site 30. In Bader, R. G., Gerard, R. D., et al., Init. Repts. DSDP, 4: Washington (U.S. Govt. Printing Office), 215-241.

Barnard, W. D., 1978. The Washington continental slope-Quaternary tectonics and sedimentation. Mar. Geol., 27:79-114.

Biju-Duval, B., Mascle, A., Montadert, L., and Wannesson, J., 1978. Seismic investigations in the Columbia, Venezuela, and Grenada basins and on the Barbados Ridge for future IPOD drilling. Geol. Mijnbouw, 57:105-116.

Biscaye, P. E., 1965. Mineralogy and sedimentation of Recent deepsea clay in the Atlantic Ocean and adjacent seas and oceans. Geol. Soc. Am. Bull., 76:803-832.

Bouma, A. H., 1962. Sedimentology of Some Flysch Deposits: Amsterdam, Holland (Elsevier Publ. Co.).

Carlson, P. R., and Nelson, C. H., 1969. Sediments and sedimentary structures of the Astoria submarine canyon-fan system, northeast Pacific. J. Sed. Petrol., 39:1269-1282.

Case, J. E., and Holcombe, T. L., 1975. Preliminary geologic-tectonic and bathymetric maps of the Caribbean region. U.S. Geol. Surv. Open File Rep. 75-146.

Chase, R. L., and Bunce, E. T., 1969. Underthrusting of the eastern margin of the Antilles by the floor of the western North Atlantic Ocean, and the origin of the Barbados Ridge. J. Geophys. Res., $74: 1413-1420$.

Christman, R. A., 1953. Geology of St. Bartholomew, St. Martin, and Anguilla, Lesser Antilles. Geol. Soc. Am. Bull., 64:65-96.

Damuth, J. E., 1975. Echo character of the western equatorial Atlantic floor and its relationship to the dispersal and distribution of terrigenous sediments. Mar. Geol., 18:17-45.

, 1977. Late Quaternary sedimentation in the western equatorial Atlantic. Geol. Soc. Am. Bull., 88:695-710.

Edgar, N. T., Saunders, J. B., et al., 1973a. Init. Repts. DSDP, 15: Washington (U.S. Govt. Printing Office).

1973b. Site 148. In Edgar, N. T., Saunders, J. B., et al., Init. Repts. DSDP, 15: Washington (U.S. Govt. Printing Office), 217-275.

Eisma, D., Van der Gaast, S. J., Martin, J. M., and Thomas, A. J., 1978. The Orinocon Delta-turbidity, mineralogy, and elementary composition. Netherlands J. Sea Res., 12(2):224-251.

Embley, R. W., and Langseth, M. G., 1977. Sedimentation processes on the continental rise of northeastern South America. Mar. Geol., 25:279-297.

Fink, L. K., Jr., 1972. Bathymetric and geologic studies of the Guadeloupe region, Lesser Antilles island arc. Mar. Geol., 12:267-288.

Fox, P. J., and Heezen, B. C., 1975. Geology of the Caribbean crust. In Nairn, A. F. M., and Stehli, F. G. (Eds.), The Ocean Basins and Margins-The Gulf of Mexico and the Caribbean (Vol. 3): New York (Plenum Press), 421-466.

Fox, P. J., Schreiber, E., and Heezen, B. C., 1971. The geology of the Caribbean crust-Tertiary sediments, granitic and basic rocks from the Aves Ridge. Tectonophysics, 12:89-109.

Griffin, J. J., and Goldberg, E. D., 1969. Recent sediments of Caribbean Sea. In McBirney, A. R. (Ed.), The Tectonic Relations of Northern Central American and the Western Caribbean-the Bonacca Expedition. Am. Assoc. Pet. Geol. Mem., 11.

Griffin, J. J., Windom, H., and Goldberg, E. D., 1968. The distribution of clay minerals in the World Ocean. Deep-Sea Res., 15: 433-459.

Grunevald, H., 1965. Géologie de la Martinique. Mémoires Pour Servir a l'Explication de la Carte Géologique Detaillée de la France: Paris (Imprimerie Nationale).

Hayes, D. E., 1974. Continental margin of western South America. In Burk, C. A., and Drake, C. L. (Eds.), The Geology of Continental Margins: New York (Springer-Verlag), pp. 581-590.

Hein, J. R., Scholl, D. W., and Gutmacher, C. E., 1976. Neogene clay minerals of the far NW Pacific and southern Bering Sea-sedimentation and diagenesis. In Bailey, S. W. (Ed.), Proc. Internat. Clay Conf. (Mexico City, Mexico, July 16-23, 1975). Assoc. Internationale pour l'étude des argiles: Wilmette, Illinois (Applied Publishers Ltd.), pp. 71-80. 
Heezen, B. C., and Rawson, M., 1977. Visual observations of the sea floor subduction line in the Middle America Trench. Science, 196: 423-426.

Hussong, D. M., Uyeda, S., et al., 1982. Init. Repts. DSDP, 60: Washington (U.S. Govt. Printing Office).

Johnson, D. A., and Driscoll, A. H., 1975. Descriptions of WHOI sediment cores (Vols. I-IV). Woods Hole Oceanographic Institution Technical Report No. 75-8. Woods Hole, Massachusetts.

Keller, G. H., Lambert, D. N., Bennett, R. H., and Rucker, J. B., 1972. Mass physical properties of Tobago Trough sediments. 6th Conf. Geol. del Caribe, Margarita, Venezuela, pp. 405-409.

Krissek, L. A., Scheidegger, K. F., and Kulm, L. D., 1980. Surface sediments of the Peru-Chile continental margin and the Nazca Plate: textural and geochemical characteristics and their controls. Geol. Soc. Am. Bull., 91:321-331.

Kulm, L. D., and Scheidegger, K. F., 1979. Quaternary sedimentation on the tectonically active Oregon continental slope. In Doyle, L. J., and Pilkey, O. H., (Eds.), Geology of Continental Slope. Soc. Econ. Paleontol. Mineral. Spec. Publ., 27:247-264.

Kulm, L. D., Schrader, H., Resig, J. M., Thornburg, T. M., Masias, A., and Johnson, L., 1981. Late Cenozoic carbonates on the Peru continental margin: lithostratigraphy, biostratigraphy, and tectonic history. In Kulm, L. D., Dymond, J., Dasch, E. J., and Hussong, D. H. (Eds.), Nazca Plate: Crustal Formation and Andean Convergence. Geol. Soc. Am. Mem. 154:469-508.

Lewis, J. F., and Robinson, E., 1976. A revised stratigraphy and geological history of the Lesser Antilles. Trans. 7th Caribbean Conf. Guadeloupe, pp. 339-344.

Lundberg, N., 1983. Development of forearcs of intraoceanic subduction zones. Tectonics, 2:51-61.

McCoy, F. W., 1969. Bottom currents in the western Atlantic Ocean between the Lesser Antilles and the Mid-Atlantic Ridge. Deep-Sea Res., 16:179-184.

McGeary, D. F. R., and Damuth, J. E., 1973. Postglacial iron-rich crusts in hemipelagic deep-sea sediment. Geol. Soc. Am. Bull., 84:1201-1212.

Marlow, M. S., Garrison, L. E., Martin, R. G., Trumbull, J. V. A., and Cooper, A. K., 1974. Tectonic transition zone in the northeastern Caribbean. J. Res. U.S. Geol. Surv., 2(3):289-302.

Martin-Kaye, P. H. A., 1959. The Geology of the Leeward and British Virgin Islands: St. Lucia (Voice Publishing Co.).

1969. A summary of the geology of the Lesser Antilles. Overseas Geol. Min. Resour., 10:172-206.

Mascle, A., Lajat, D., and Nely, G., 1979. Sediment deformation linked to subduction and to argillokinesis in the southern Barbados Ridge from multichannel seismic surveys. Internat. Symp. Geodyn. Southwest Pacific (Nov. 1976): Paris (Technip), pp. 327-344.

Metcalf, W. G., 1976. Water exchange between the Atlantic Ocean and the Caribbean Sea. CICAR II Symp. Progress in Marine Research in the Caribbean and Adjacent Regions, FAO, Caracas, pp. 63-102.

Milliman, J. D., and Bornhold, B. D., 1973. Discussion: Peak height versus peak intensity analysis of X-ray diffraction data. Sedimentology, 20:445-448.

Minster, J. B., and Jordan, T. H., 1978. Present-day plate motions. J. Geophys. Res., 83:5331-5354.

Moore, J. C., Biju-Duval, B., Bergen, J. A., Blackington, G., Claypool, G. E., et al., 1982. Offscraping and underthrusting of sediment at the deformation front of the Barbados Ridge: Deep Sea Drilling Project Leg 78A. Geol. Soc. Am. Bull., 93:1065-1077.

Moore, J. C., Watkins, J. S., McMillen, K. J., Bachman, S. B., and Leggett, J. K., et al., 1982. Facies belts of the Middle America Trench and forearc region, southern Mexico-results from Leg 66 DSDP. In Leggett, J. K. (Ed.), Thench-Forearc Geology: Sedimentation and Tectonics on Modern and Ancient Active Plate Margins. Geol. Soc. London Spec. Publ. 10: London (Blackwells), pp. 77-94.

Müller, G., and Gastner, M., 1971. The “Karbonate Bombe," a simple device for determination of the carbonate content in sediments, soils, and other materials. Neues Jahrb. Mineral. Montash., 10: 466-469.

Nagle, F., 1972. Rocks from seamounts and escarpments on the Aves Ridge. 6th Conf. Geol. del Caribe, Caracas, Venezuela, pp. 409-413.
Nagle, R., Erlich, R. N., and Canovi, C. J., 1978. Caribbean dredge haul compilation-summary and implication. In MacGillavry, H. J., and Beets, D. J. (Eds.). 8th Caribbean Geol. Conf. Geol. Mijnbouw, 57:267-270.

Newell, R. E., Kidson, J. W., Vincent, G., and Boer, G. J., 1972. The General Circulation of the Tropical Atmosphere (Vol. 1): Cambridge (Cambridge MIT Press).

Nota, D. J. G., 1958. Sediments of the Western Guiana Shelf-Reports of the Orinoco Shelf Expedition, 1952-1953 (Vol. II): Wageningen, Holland (H. Veenman and Zonen).

Peter, G., and Westbrook, G. K., 1976. Tectonics of the southwestern North Atlantic and Barbados Ridge complex. Am. Assoc. Pet. Geol. Bull., 60:1078-1106.

Piper, D. J. W., von Huene, R., and Duncan, J. R., 1973. Late Quaternary sedimentation in the active eastern Aleutian Trench. Geology, 1:19-22.

Pudsey, C. J., and Reading, H. G., 1982. Sedimentology and structure of the Scotland Group, Barbados. In Leggett, J. K. (Ed.), TrenchForearc Geology: Sedimentation and Tectonics on Modern and Ancient Active Plate Margins. Geol. Soc. London Spec. Publ. 10: London (Blackwells), pp. 291-308.

Robinson, E., and Jung, P., 1972. Stratigraphy and age of marine rocks, Carriacou, West Indies. Am. Assoc. Pet. Geol. Bull., 56: 114-127.

Rosato, V. J., and Kulm, L. D., 1981. Clay mineralogy of the Peru continental margin and adjacent Nazca Plate: implications for provenance, sea level changes, and continental accretion. In Kulm, L. D., Dymond, J., Dasch, E. J., and Hussong, D. H. (Eds.), Nazca Plate: Crustal Formation and Andean Convergence. Geol. Soc. Am. Mem., 154:545-568.

Ross, D. A., 1971. Sediments of the northern Middle America Trench. Geol. Soc. Am. Bull., 82:303-322.

Scholl, D. W., Christensen, M. N., von Huene, R., and Marlow, M. S., 1970. Peru-Chile Trench sediments and sea-floor spreading. Geol. Soc. Am. Bull., 81:1339-1360.

Scholl, D. W., and Marlow, M. S., 1974. Sedimentary sequences in modern Pacific trenches and the deformed circum-Pacific eugeosyncline. In Dott, R. H., and Shaver, R. H. (Eds.), Modern and Ancient Geosynclinal Sedimentation. Soc. Econ. Paleontol. Mineral. Spec. Publ., 19:193-211.

Scholl, D. W., Marlow, M. S., and Cooper, A. K., 1977. Sediment subduction and offscraping at Pacific margins. In Talwani, M., and Pitman, W. C. III (Eds.), Island Arcs, Deep Sea Trenches, and Backarc Basins. Washington (Am. Geophys. Union, Maurice Ewing Series), 1:199-210.

Scholl, D. W., Vallier, T. L., and Stevenson, A. J., 1982. Sedimentation and deformation in the Amlia Fracture Zone sector of the Aleutian Trench. Mar. Geol., 48:105-134.

Schweller, W. J., and Kulm, L. D., 1978. Depositional patterns and channelized sedimentation in active eastern Pacific trenches. In Stanley, D. J., and Kelling, G. (Eds.), Sedimentation in Submarine Canyons, Fans, and Trenches: Stroudsburg, Pennsylvania (Dowden, Hutchinson, and Ross), pp. 311-324.

Shephard, L. E., and McMillen, K. J., 1982. Sedimentation rates of the southern Mexico continental margin, DSDP Leg 66. In Moore, J. C., Watkins, J. S., et al., Init. Repts. DSDP, 66: Washington (U.S. Govt. Printing Office), 445-451.

Sigurdsson, H., Sparks, R. J. S., Carey, S., and Huang, T. C., 1980. Volcanogenic sedimentation in the Lesser Antilles Arc. J. Geol., 88:523-540.

Speed, R. C., 1981. Geology of Barbados: implication for an accretionary origin. Oceanol. Acta, 4 (Suppl.):259-265.

Supko, P., Ross, D. A., and Neprochnov, Y. P., 1978. Introduction and explanatory notes, Leg 42B, Deep Sea Drilling Project. In Ross, D. A., Neprochnov, Y. P., et al., Init. Repts. DSDP, 42, Pt. 2: Washington (U.S. Govt. Printing Office), 3-15.

Tomblin, J. F., 1975. The Lesser Antilles and Aves Ridge. In Nairn, A. E. M., and Stehli, F. G. (Eds.), The Ocean Basins and MarginsThe Gulf of Mexico and the Caribbean (Vol. 3): New York (Plenum Press), 467-500.

Underwood, M. B., and Bachman, S., 1982. Sedimentary facies associations within subduction complexes. In Leggett, J. K. (Ed.), Trench- 


\section{A. WRIGHT}

Forearc Geology: Sedimentation and Tectonics on Modern and Ancient Active Plate Margins. Geol. Soc. London Spec. Publ. 10: London (Blackwells), pp. 537-550.

Underwood, M. B., Bachman, S. B., and Schweller, W. J., 1980. Sedimentary processes and facies association within trench and trenchslope settings. In Field, M. E., et al. (Eds.), Quaternary Depositional Environments on the Pacific Continental Margin. Soc. Econ. Paleontol. Mineral., Pacific Section: 211-229.

Underwood, M. B., and Karig, D. E., 1980. Role of submarine canyons in trench-slope sedimentation. Geology, 8:432-436.

van Andel, Tj. H., 1975. Mesozoic/Cenozoic calcite compensation depth and the global distribution of calcareous sediments. Earth Planet. Sci. Lett., 26:187-194.

von Huene, R., Aubouin, J., et al., 1980. Leg 67, the DSDP MidAmerican transect off Guatemala. Geol. Soc. Am. Bull., 91: 421-432.

Westbrook, G. W., 1975. The structure of the crust and upper mantle in the region of Barbados and the Lesser Antilles. Geophys. J. R., 43:201-242.
1982. The Barbados Ridge Complex-tectonics of a mature forearc system. In Leggett, J. K. (Ed.), Trench-Forearc Geology: Sedimentation and Tectonics on Modern and Ancient Active Plate Margins. Geol. Soc. London Spec. Publ. 10: London (Blackwells), pp. 275-290.

Westercamp, D., 1977. Evolution des suies volcaniques de Martinique (FWI) et des arcs insulairs des Petites Antilles dans les contexts structural: Trans. 8th Caribbean Geol. Conf., Willemstad, pp. 227-228.

Williams, E. McB., 1977. R/V Trident Cruise summaries CY1962 through CY1971. University of Rhode Island Graduate School of Oceanography Technical Report Reference No. 77-4. Kingston, Rhode Island.

Worsley, T. R., and Davies, T. A., 1979. Cenozoic sedimentation in the Pacific Ocean-steps toward a quantitative evaluation. J. Sed. Petrol., 49:1131-1148.

Date of Initial Receipt: December 17, 1982

Date of Acceptance: June 6, 1983 